\title{
Remeshing criteria and proper error representations for goal oriented $h$-adaptivity ${ }^{\star}$
}

\author{
Pedro DÍEZ ${ }^{1, a}$ and Giovanni CALDERÓN ${ }^{\mathrm{a}, \mathrm{b}}$ \\ ${ }^{\mathrm{a}}$ LaCàN, Departament de Matemàtica Aplicada 3, \\ Universidad Politécnica de Cataluña, \\ Jordi Girona 1, E-08011 Barcelona, Spain. \\ tel. +34934017240 fax. +34934011825 \\ ${ }^{\mathrm{b}}$ Departamento de Matemáticas, Facultad de Ciencias, Universidad de Los Andes, \\ La Hechicera, Mérida, Venezuela
}

\begin{abstract}
A key ingredient in $h$-adaptivity pertains to the transformation of output data from a given error estimator into input data, usually in the form of an element-size distribution, that needs to be supplied to a mesh generator.

This paper analyzes the different possibilities of defining remeshing criteria in the framework of goal oriented adaptivity. In standard energy norm driven adaptivity, the optimal mesh is clearly obtained if the local error distribution is uniform. The goal-oriented paradigm introduces new difficulties associated with the different possibilities for the spatial error representation and the signs of the local error contributions.
\end{abstract}

A nodal error representation is introduced in order to improve the communication with the automatic mesh generation tool, precluding the transfer of information from elements to nodes.

Numerical experiments demonstrate the ability of the introduced remeshing strategies to drive efficient adaptive procedures and to control the error in quantities of interest. The results of the numerical tests fit the expected properties of the different remeshing strategies.

Key words: goal oriented adaptivity, remeshing criteria, error in quantities of interest, optimal error representation 


\section{INTRODUCTION}

The paradigm of adaptivity in computational mechanics is to design the less costly mesh producing a numerical solution fulfilling the accuracy prescriptions. The main ingredients in any adaptive procedure are an error estimator and a mesh generator. The error estimate is required to decide if the adaptive loop must be stopped and to locate the zones in the domain where the elements must be concentrated (where the contributions to the error are large). The mesh generator produces the mesh with the required element size in every part of the domain, as determined after the error assessment. The remeshing criterion is the expression that translates the output of the error estimator into a spatial distribution of the optimal element size for the new mesh, which is the input of the mesh generator.

In the context of adaptivity based in the energy norm, attention has been devoted to remeshing criteria yielding the element size as a function of the local error, see [1-4]. In the framework of goal oriented adaptivity, where the aim is to control the error in some quantity of interest instead of in the energy norm, each author follows his or her own recipe, based often on heuristic considerations and without any claim on the optimality of the designed mesh

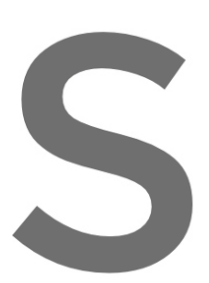
$[5-7]$. Following a different approach,
gence rates of some adaptive procedu
literature, see $[8-11]$. In these yor ks,
refining the initial mesh, keeping the n
adaptive process.
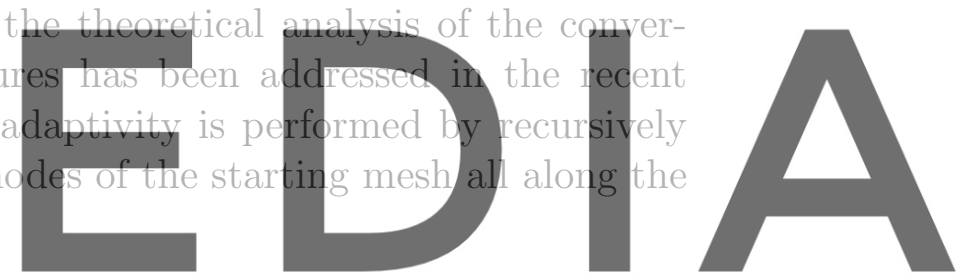

The particularities associated with remeshing criteria in goal oriented adap-

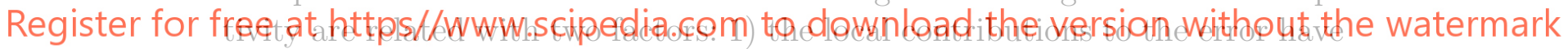
in general different sign and 2) the spatial error distribution is not unique.

The different sign of the local contributions to the error means that, in some parts of the domain, refining the discretization decreases the error in the output of interest while in other zones mesh refinement has the opposite effect. In that sense, one could expect balancing the errors coming from different zones to control the final error. Nevertheless, a remeshing strategy based on balancing the error contributions would be unstable, requiring a high accuracy in both the error estimate and the automatic mesh generator. In practice, it is much more sensible to use the absolute values of the error and to refine all

* Partially supported by Ministerio de Educación y Ciencia, Grants DPI2004-03000 and CGL2004-06171-C03-01/CLI and by CDCHT-ULA, Venezuela C-1373-06-05-B

Email addresses: pedro.diez@upc.es (Pedro DÍEZ), giovanni@ula.ve

(Giovanni CALDERÓN).

URL: www-lacan.upc.es (Pedro DÍEZ ).

1 Corresponding author. 
the zones where the error contributions are large (in absolute value), independently of their sign and not trying to balance the large error contributions with opposite sign. Obviously, this kind of strategy leads to very conservative meshes, with a large number of elements, especially if the spatial error distribution has local contributions with different sign that compensate each other.

The lack of uniqueness of the error distribution arises from the different possible error representations. The error in the quantity of interest is described by different equivalent expressions resulting in different local error distributions. Obviously, if the remeshing criterion is based on the absolute values of the local contribution to the error, the spatial error distributions preferred should have all the local contributions with the same sign. If this is not possible, this condition can be relaxed to obtain most of the local contributions with the same sign, in order to minimize the effect in the resulting mesh of using absolute values.
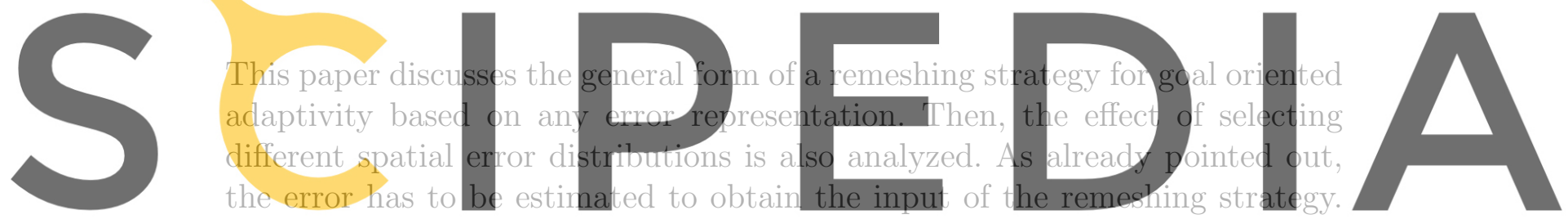

Thus, the errors in the error assessment introduced by the error estimation

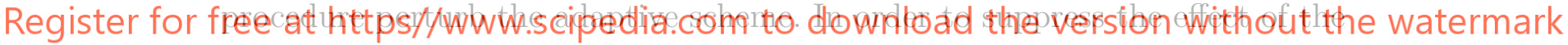
error estimate, in this work the error is not estimated but computed with an overkill mesh (when the exact solution is not available). Obviously, this strategy cannot be used in practical applications because of its computational cost but it simplifies the analysis of the adaptive procedure, isolating its effect in the resulting adapted solution.

The remainder of the paper is structured as follows. In section 2 the problem is stated in a general framework, introducing the notation. Section 3 develops the remeshing criteria for goal oriented $h$-adaptivity starting from the basic assumptions on the local convergence rate and the expected error distribution (somehow uniform). In fact, the uniform error distribution is optimal in the sense that it produces meshes with the least number of elements, as stated in section 4. Section 5 explores the different choices for the spatial error distribution and their consequences in the remeshing process. 


\section{PROBLEM STATEMENT}

\subsection{Model problem}

The unknown function $u$ is the solution of a boundary-value problem defined in $\Omega \subset \mathbb{R}^{d}$, a bounded $d$-dimensional open domain, where $d$ is equal to 1,2 or 3. The weak form of the problem reads: find $u \in \mathcal{V}$ such that

$$
a(u, v)=\ell(v), \text { for all } v \in \mathcal{V}_{0},
$$

where the functional spaces $\mathcal{V}$ and $\mathcal{V}_{0}$ differ by the values that the functions take on the Dirichlet part of the boundary: functions in $\mathcal{V}$ fulfil the Dirichlet boundary conditions and functions in $\mathcal{V}_{0}$ their homogeneous counterpart.

For elliptic self-adjoint problems, the bilinear form $a(\cdot, \cdot)$ is symmetric and positive definite. It is worth noting that in the following developments we do not restrict ourselves to this case. However, the numerical tests shown in section 6 concern such particular problems.
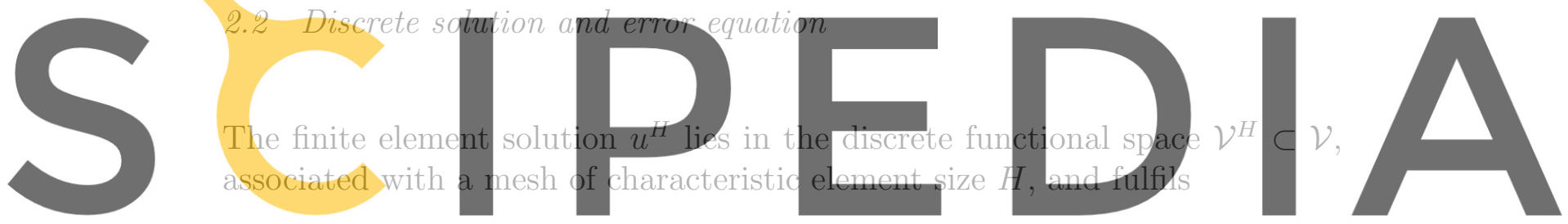

$a\left(u^{H}, v\right)=\ell(v)$, for all $v \in \mathcal{V}_{0}^{H} \subset \mathcal{V}_{0}$.

(2)

Register for free at https//www.scipedia.com to download the version without the watermark

The error of the numerical solution, $e:=u-u^{H}$, lies in $\nu_{0}$ and fulfils the following residual equation

$$
a(e, v)=\ell(v)-a\left(u^{H}, v\right)=: R^{P}(v), \text { for all } v \in \mathcal{V}_{0},
$$

where the residual in equation $(1), R^{P}(\cdot)$, is introduced.

\subsection{Output of interest, dual problem and error representation}

In the context of goal-oriented adaptivity, the aim is to assess the error of some output of interest. In the following, we restrict ourselves to the case of a linear output. Thus, the quantity of interest is represented by a linear functional $J(\cdot)$ and the goal is to assess and control the output error, $J(e)$. In order to express the error in the output of interest in terms of energy products, an 
auxiliary dual (or adjoint) problem is introduced. The dual problem consists on finding $\varphi$ in $\mathcal{V}_{0}$ such that

$$
a(v, \varphi)=J(v), \text { for all } v \in \mathcal{V}_{0} .
$$

Then, setting $v=e$ in (4), the following error representation is readily found

$$
J(e)=a(e, \varphi)=R^{P}(\varphi) .
$$

Note that the Galerkin orthogonality property holds and therefore for all $v^{H}$ in $\mathcal{V}_{0}^{H}$,

$$
a\left(e, v^{H}\right)=0 .
$$

Consequently, the error representation (5) may be modified introducing an arbitrary function $v^{H}$

$$
J(e)=a\left(e, \varphi-v^{H}\right)=R^{P}\left(\varphi-v^{H}\right) .
$$

In particular, letting $v^{H}$ be the solution of the dual problem (4) in $\mathcal{V}_{0}^{H}$, that is $v^{H}=\varphi^{H}$, the error in the output is expressed in terms of a combination of
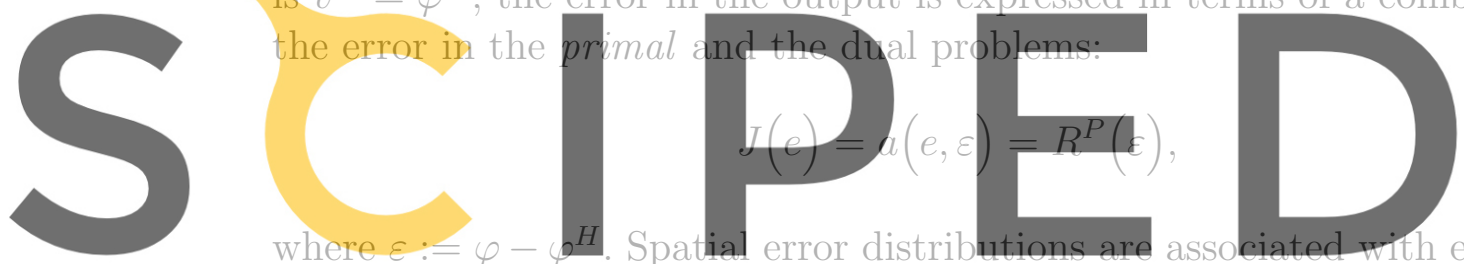

representation. These allow assessing the contribution of every zone of the

\subsection{Spatial error distribution}

The local error contributions are usually associated with the elements of the mesh inducing $\mathcal{V}_{0}^{H}$. The natural restriction to every element $\Omega_{k}, k=1, \ldots, \mathrm{n}_{\mathrm{el}}$, $\mathrm{n}_{\mathrm{e} 1}$ being the number of elements in the mesh of characteristic size $H$, of the integral forms in the error representation (7) (or the particular case (8)) yields the element by element error distribution:

$$
J(e)=\sum_{k=1}^{\mathrm{n}_{\mathrm{e} 1}} a_{k}\left(e, \varphi-v^{H}\right)=\sum_{k=1}^{\mathrm{n}_{\mathrm{e} 1}} R_{k}^{P}\left(\varphi-v^{H}\right),
$$

where $a_{k}(\cdot, \cdot), \ell_{k}(\cdot)$ and thus $R_{k}^{P}(\cdot):=\ell_{k}(\cdot)-a_{k}\left(u^{H}, \cdot\right)$ are the contributions, computed in $\Omega_{k}$ to the global quantities $a(\cdot, \cdot), \ell(\cdot)$ and $R_{k}^{P}(\cdot)$, respectively. 
Note that the local quantities $a_{k}\left(e, \varphi-v^{H}\right)$ and $R_{k}^{P}\left(\varphi-v^{H}\right)$ represent different elementary contributions to the error and that they are not necessarily positive.

Moreover, the function $v^{H}$ is arbitrarily taken in $\mathcal{V}_{0}^{H}$ and every choice for $v^{H}$ induces a different error distribution. As it is discussed in the following, the natural choice $v^{H}=\varphi^{H}$ is often a good option.

\section{A GENERAL FRAMEWORK FOR REMESHING CRITERIA}

\subsection{Goals and notation}

Once the error is assessed, a key ingredient in the adaptive procedure is the remeshing criterion. The remeshing criterion takes as input the error distribution and produces the information required to build up a new mesh. If the remeshing criterion is properly derived, the new mesh should provide an approximate solution satisfying the accuracy requirements at a minimum computational cost. From a practical viewpoint, the remeshing criterion is produced
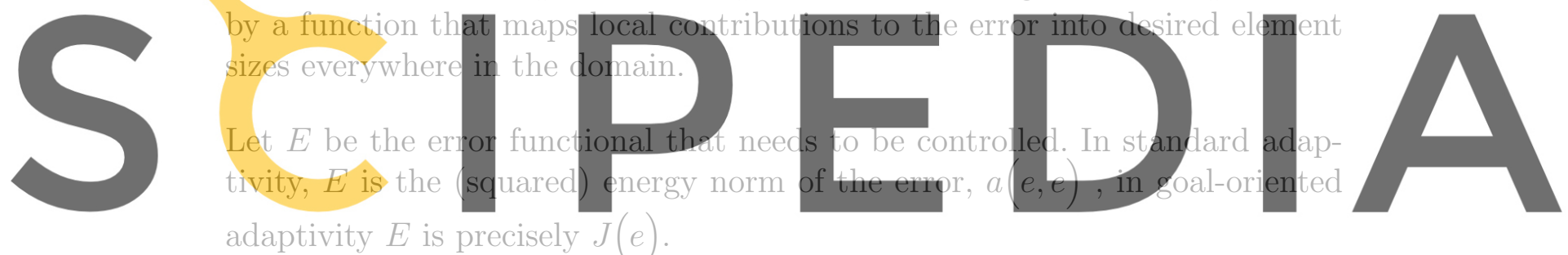

Register for free at https//www.scipedia.com to download the version without the watermark

The quantity $E$ is decomposed into elementary contributions $E_{k}$, for $k=$ $1, \ldots, \mathrm{n}_{\mathrm{e} 1}$ :

$$
E=\sum_{k=1}^{\mathrm{n}_{\mathrm{e} 1}} E_{k}
$$

Each element in the mesh is denoted by $\Omega_{k}$ and the size of this element is denoted by $H_{k}$. If the elements in the mesh are sufficiently regular (not too distorted), the size of each element is taken as

$$
H_{k}=\left[\text { meas } \Omega_{k}\right]^{1 / d}
$$

From now on, quantities that are defined in the new mesh are denoted with the hat symbol $(\hat{\bullet})$. For example, the number of elements and characteristic element size are denoted by $\hat{\mathrm{n}}_{\mathrm{e} 1}$ and $\widehat{H}$. The local element size in the new mesh should be denoted by $\widehat{H}_{\hat{k}}$ for $\hat{k}=1, \ldots, \hat{\mathrm{n}}_{\mathrm{e} 1}$. Nevertheless, the remeshing criterion furnishes the element size in the new mesh for the elements located in the position of the former element $\Omega_{k}$. Thus, it is more convenient to use 
the notation $\widehat{H}_{k}$ to denote the size of the elements in the new mesh associated with $\Omega_{k}$. Note that each value of $\widehat{H}_{k}$ corresponds to different elements in the new mesh, that is to several values of $\widehat{H}_{\hat{k}}$.

The goal is then to derive an expression for $\widehat{H}_{k}$ as a function of $E_{k}$ and $H_{k}$ such that the new mesh meets the accuracy requirements at the minimum cost. This remeshing criterion is expected to produce the mesh size distribution for the optimal mesh.

\subsection{Assumptions required: local convergence rate, optimal error distribution}

Deriving a remeshing criterion requires some further assumptions, both in the local convergence of the solutions and in the error distribution on the optimal mesh.

First, an a priori estimate is needed for the local contributions to the error. The usual form for the local a priori error estimates, both in energy norm or in other quantities of interest, reads
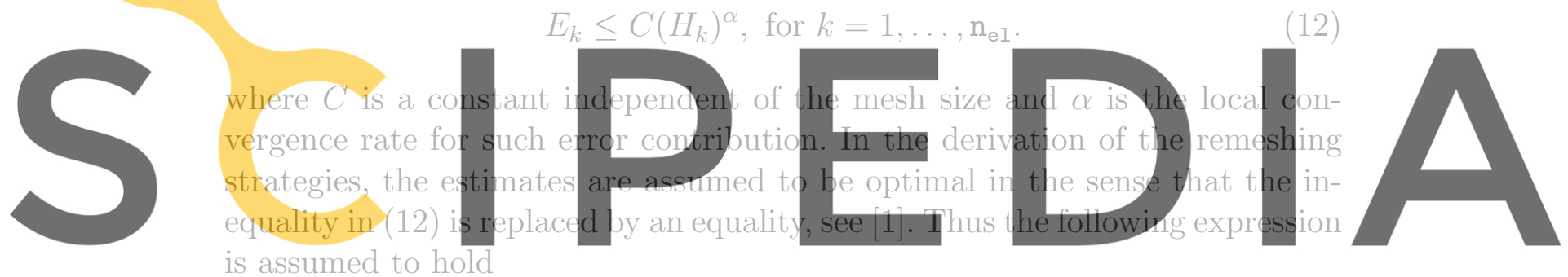

Register for free at https//www.scipedia.jam to

The value of $\alpha$ is derived from the usual energy norm a priori error estimates because the error contributions $E_{k}$ are defined from an error representation involving energy products of error quantities, as described in equation (8). This convergence rate is assumed to hold also for the new mesh, that is

$$
\widehat{E}_{\hat{k}}=C\left(\widehat{H}_{\hat{k}}\right)^{\alpha}, \text { for } \hat{k}=1, \ldots, \hat{\mathrm{n}}_{\mathrm{el}} .
$$

Second, an assumption must be done on the error distribution in the optimal mesh. Usually this results on establishing some desired uniformity on the error distribution.

For instance, the new mesh is sought such that all the elementary contributions are equal, that is all $\widehat{E}_{\hat{k}}$, for $\hat{k}=1, \ldots, \hat{\mathrm{n}}_{\mathrm{e} 1}$, are equal and do not depend on $\hat{k}$. In fact this is the remeshing criterion proposed by Li and Bettess [1,2] for adaptivity in the energy norm. This criterion is optimal in the sense that it 
furnishes the meshes with fewer elements and with the prescribed value of the energy norm of the error. It is proved in the next section that this criterion is also optimal for goal oriented adaptivity.

The error sought in the new mesh, $\widehat{E}$ is given by the user. Thus, this remeshing criterion requires

$$
\widehat{E}_{\hat{k}}=\widehat{E} / \hat{\mathrm{n}}_{\mathrm{el}}, \text { for } \hat{k}=1, \ldots, \hat{\mathrm{n}}_{\mathrm{el}} \text {. }
$$

Note that $\hat{\mathrm{n}}_{\mathrm{el}}$ is not known a priori but it is going to be predicted using one additional assumption.

\subsection{Derivation of the remeshing criterion}

With the ingredients listed in the previous section the expression for the remeshing criterion is readily derived. The constant $C$ corresponding to element $\Omega_{k}$ is isolated from equation (13) and it is found to be

$$
C=E_{k} /\left(H_{k}\right)^{\alpha}
$$

Replacing $C$ in (14), using (15) and considering that, for the new elements located in $\Omega_{k}, \widehat{H}_{\hat{k}}$ is denoted $\widehat{H}_{k}$, one gets
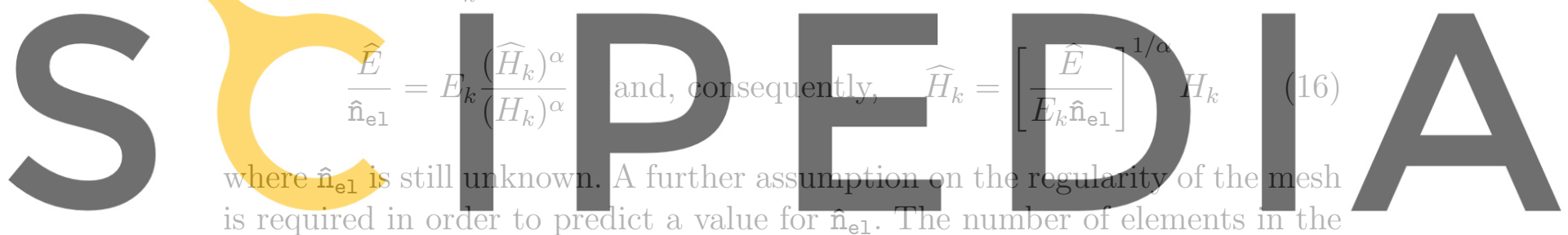

Register for free at https//www.scipedia.com to download the version without the watermark

to be $\left|\frac{H_{k}}{\widehat{H}_{k}}\right|$. Note that this is a particular case of (11) and it is equivalent to

assume that the aspect ratio of the elements in both the current mesh and the new mesh is similar in every part of the domain. This hypothesis is found to be very accurate in practice. Thus, the following expression for $\hat{n}_{\mathrm{el}}$ follows

$$
\hat{\mathrm{n}}_{\mathrm{el}}=\sum_{k=1}^{\mathrm{n}_{\mathrm{el}}}\left[\frac{H_{k}}{\widehat{H}_{k}}\right]^{d} \text {. }
$$

Introducing (16) in (17) yields

$$
\hat{\mathrm{n}}_{\mathrm{el}}=\sum_{k=1}^{\mathrm{n}_{\mathrm{e} 1}}\left[\frac{E_{k} \hat{\mathrm{n}}_{\mathrm{el}}}{\widehat{E}}\right]^{d / \alpha}=\frac{\widehat{\mathrm{n}}_{\mathrm{el}}^{d / \alpha}}{\widehat{E}^{d / \alpha}} \sum_{k=1}^{\mathrm{n}_{\mathrm{e} 1}}\left(E_{k}\right)^{d / \alpha}
$$

and $\hat{\mathrm{n}}_{\mathrm{el}}$ is isolated from the previous equation

$$
\hat{\mathrm{n}}_{\mathrm{el}}=\left[\frac{1}{\widehat{E}^{d / \alpha}} \sum_{k=1}^{\mathrm{n}_{\mathrm{el}}}\left(E_{k}\right)^{d / \alpha}\right]^{\alpha /(\alpha-d)} .
$$


It is worth noting that the expression (18) is computable once the error in the current mesh is assessed (all $E_{k}$ are known) and the target error $\widehat{E}$ is prescribed.

In the developments of this section it has been implicitly assumed that the local error contributions are all positive or, more generally, have all the same sign, see for instance (15). As mentioned above, this assumption is very conservative, especially if the error distribution is such that the local error contributions have different sign and compensate each other. Nevertheless, from a practical viewpoint, exploiting the different signs of the error contribution in order to balance them is not realistic. It is much easier to use a proper error representation.

\section{OPTIMALITY OF UNIFORM ERROR DISTRIBUTION}

In the previous section it has been claimed that the uniform error distribution given by (15) is optimal in the sense that it produces meshes with fewer elements. This claim is proved for energy norm based adaptivity in [1,2] and the same rationale is also valid in the context of goal oriented adaptivity.
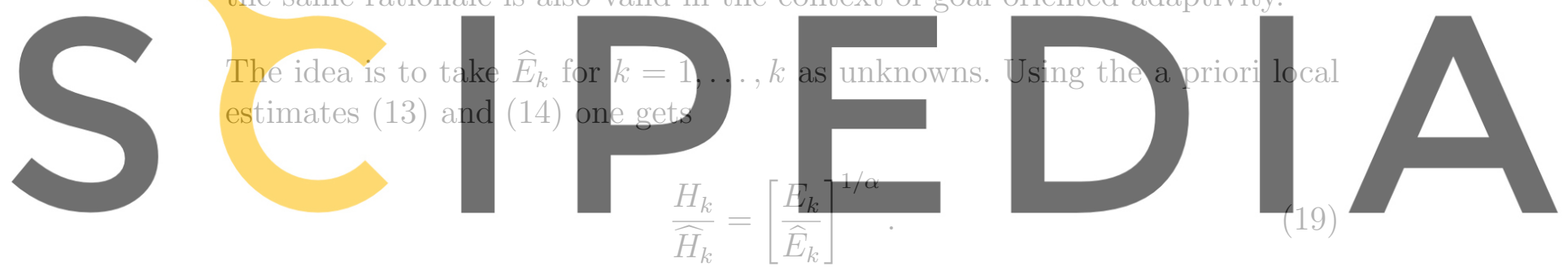

Register for free at https//www.scipedia.com to download the version without the watermark Replacing (19) in (17) yields

$$
\hat{\mathrm{n}}_{\mathrm{el}}=\sum_{k=1}^{\mathrm{n}_{\mathrm{el}}}\left[\frac{E_{k}}{\widehat{E}_{k}}\right]^{d / \alpha}
$$

The goal is then to find $\widehat{E}_{k}$ for $k=1, \ldots, k$ minimizing $\hat{\mathrm{n}}_{\mathrm{el}}$ subject to the constraint

$$
\widehat{E}=\sum_{\widehat{k}=1}^{\hat{\mathrm{n}}_{\mathrm{e} 1}} \widehat{E}_{\widehat{k}}
$$

Note that using the mesh regularity assumption this is equivalent to

$$
\widehat{E}=\sum_{k=1}^{\mathrm{n}_{\mathrm{e} 1}} \widehat{E}_{k}\left[\frac{H_{k}}{\widehat{H}_{k}}\right]^{d}=\sum_{k=1}^{\mathrm{n}_{\mathrm{e} 1}} \widehat{E}_{k}\left[\frac{E_{k}}{\widehat{E}_{k}}\right]^{d / \alpha}=\sum_{k=1}^{\mathrm{n}_{\mathrm{e} 1}} \widehat{E}_{k}^{1-d / \alpha} E_{k}^{d / \alpha} .
$$

Using the Lagrange multipliers approach, minimizing (20) subject to the re- 
striction (22) is equivalent to find $\widehat{E}_{k}$ for $k=1, \ldots, k$ and $\lambda$ such that

$$
F\left(\widehat{E}_{1}, \ldots, \widehat{E}_{\mathrm{n}_{\mathrm{e} 1}}, \lambda\right):=\sum_{k=1}^{\mathrm{n}_{\mathrm{e} 1}}\left[\frac{E_{k}}{\widehat{E}_{k}}\right]^{d / \alpha}-\lambda\left(\widehat{E}-\sum_{k=1}^{\mathrm{n}_{\mathrm{e} 1}} \widehat{E}_{k}^{1-d / \alpha} E_{k}^{d / \alpha}\right)
$$

is stationary. Consequently, for every $l$ from 1 to $\mathrm{n}_{\mathrm{el}}$,

$$
\frac{\partial F}{\partial \widehat{E}_{l}}=0, \quad \text { that is } \quad E_{l}^{d / \alpha} \widehat{E}_{l}^{-d / \alpha}\left[(-d / \alpha) \widehat{E}_{l}^{-1}+\lambda(1-d / \alpha)\right]=0
$$

or, conversely,

$$
\widehat{E}_{l}=\frac{d}{\lambda(\alpha-d)} .
$$

Obviously, this requires all values of $\widehat{E}_{k}$ for $k=1, \ldots, \mathrm{n}_{\mathrm{el}}$ to be equal and proofs that the optimality criterion (15) used in the previous section is optimal in the sense that the resulting mesh is expected to have the least number of elements. This criterion and the corresponding remeshing strategy are denoted in the following by UED (Uniform Error Distribution).

Nevertheless, other optimality criteria may have other desirable properties and may also be used to derive different expressions for remeshing criteria. This
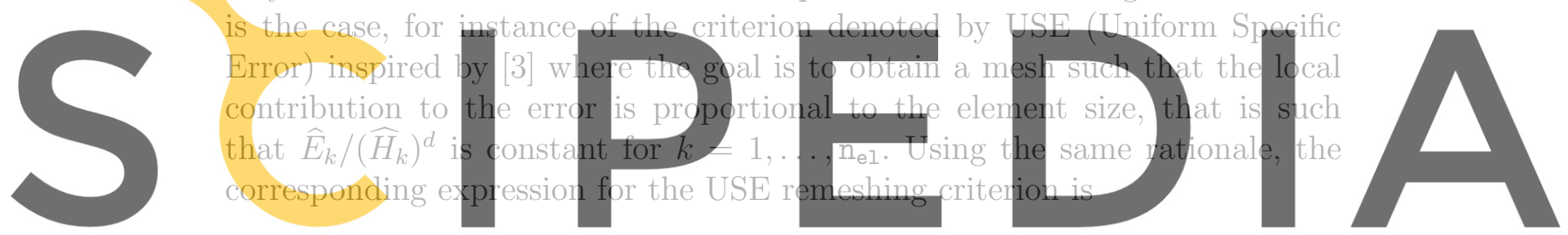

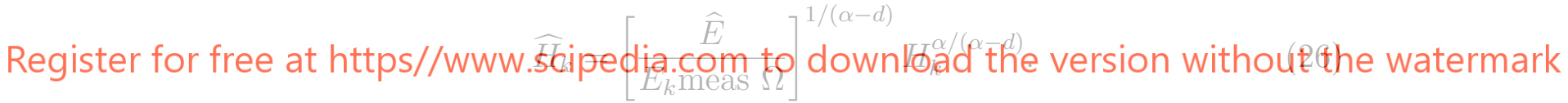

Note that the USE remeshing strategy is not optimal in the sense that it is expected to produce meshes with more elements than UED. Nevertheless, the meshes produced by the USE criterion may have other desirable features and therefore the USE criterion is also used in the examples.

\section{PROPER LOCAL ERROR REPRESENTATION AND ERROR DISTRIBUTION}

In the previous sections remeshing criteria for goal oriented adaptivity have been derived. The input data for such expressions are: the local contributions to the error, $E_{k}$ for $k=1, \ldots, \mathrm{n}_{\mathrm{el}}$, the local convergence rate, $\alpha$ and the target error, $\widehat{E}$, see for instance (16) and (18) and also (26). The target error is prescribed by the user, while the error distribution has to be assessed using 
some error estimator. It has already been mentioned that the error distribution is not unique and has to be chosen. In fact, $\alpha$ depends on this choice. This section is devoted to analyze different possible error distributions and the resulting remeshing criteria.

\subsection{Elementary error product}

The obvious choice for the spatial error distribution is $E_{k}=a_{k}(e, \varepsilon)$ that corresponds to setting $v^{H}=\varphi^{H}$ in (9), see for instance [6,5]. The local convergence rate corresponding to this distribution is assumed to be $\alpha=2 p+d, p$ being the degree of the interpolation and $d$ the space dimensions, for regions free of singularities. This is due to the fact that the energy product of the two errors $e$ and $\varepsilon$ converges with a rate double than the single energy norm of each of them. This is a priori the best choice for the error distribution because it yields the higher expectable local convergence rate, the largest value for $\alpha$.

In practice, the data entering the expressions for the remeshing criteria ((16) or (26)) has to be positive and, consequently, the actual choice is $E_{k}=\left|a_{k}(e, \varepsilon)\right|$. If the original distribution of $a_{k}(e, \varepsilon)$ has alternate signs the resulting remeshing criterion produces meshes with a number of elements much larger than needed. In fact, in these cases the sum of all $E_{k}$ is much larger than $J(e)$.

Two different approaches are proposed in order to minimize the effect of the different signs of the local error contributions. The first idea, developed in section 5.2, is based on selecting the optimal $v^{H}$ in (9). The second approach, described in section 5.3, consists on balancing the effect of the signs by using a proper factor to correct the local error contributions.

\subsection{Optimal choice for $v^{H}$}

It has been noticed in (7) that the error representation admits any function $v^{H} \in \mathcal{V}_{0}^{H}$. Using different values for $v^{H}$ does not modify the global representation of $J(e)$. Nevertheless, the local contributions to the error, $a_{k}\left(e, \varphi-v^{H}\right)$, are very different depending on the choice of $v^{H}$. Thus, in this section $v^{H}$ is selected such that the local error representation is optimal in the sense that it induces a remeshing strategy leading to a mesh with the least number of elements.

It is obvious from (18) that for the UED the optimal error representation is 
such that

$$
S:=\sum_{k=1}^{\mathrm{n}_{\mathrm{e} 1}}\left(E_{k}\right)^{d / \alpha}
$$

is minimum. Note, however, that the value of the local rate of convergence, $\alpha$, depends also on the choice of $v^{H}$. As already mentioned, $\alpha=2 p+d$ for $v^{H}=\varphi^{H}$ and $\alpha=p+d / 2$ if $v^{H}$ is selected arbitrarily (likely $\varphi-v^{H}$ does not depend on $H$ ).

Recall that, in order to preserve the stability of the remeshing process, the remeshing criterion is based on the absolute values of the local error contribution, namely

$$
E_{k}=\left|a_{k}\left(e, \varphi-v^{H}\right)\right| .
$$

Thus, the goal is to select $v^{H}$ giving the least value for the sum of some power $(d / \alpha)$ of the absolute values of the local error contributions.

The function $v^{H}$ is represented by its coefficients $c_{i}, i=1, \ldots, \mathrm{n}_{\mathrm{dof}}$ in the basis of shape functions $\left\{N_{1}, \ldots, N_{\mathrm{n}_{\text {dof }}}\right\}$ generating $\mathcal{V}_{0}^{H}$, that is

$$
v^{H}=\sum_{i=1}^{\mathrm{n}_{\mathrm{dof}}} c_{i} N_{i}
$$

Thus, finding $v^{H}$ is equivalent to finding the unknown vector $\boldsymbol{c}$ of the coefficients $c_{i}, i=1, \ldots, \mathrm{n}_{\mathrm{dof}}$. Minimizing $S$ leads generally to a nonlinear system of equations for $\boldsymbol{c}$.

The computational effort devoted to obtain $\boldsymbol{c}$ and hence $v^{H}$ is worthwhile if the distribution of local energy products, $a_{k}(e, \varepsilon)$, see section 5.1, is balanced. That is, if the local error contributions with opposite sign compensate each other and the global value of $J(e)$ is significantly lower than some of the local errors. In these cases, introducing a proper value for $v^{H}$ produces a local error distribution as homogeneous as possible and reduces most of the undesirable effects of taking absolute values in the definition of $E_{k}$.

In order to obtain such homogeneousness in the error distribution at the lowest computational cost, functional $S$ is replaced by

$$
S^{\star}:=\sum_{k=1}^{\mathrm{n}_{\mathrm{e} 1}}\left(E_{k}\right)^{2}
$$

Then, the problem is formulated as a classical least squares fitting and finding $\boldsymbol{c}$ is equivalent to solving the corresponding normal equations. The local error contribution is rewritten as

$$
E_{k}=a_{k}(e, \varphi)-\sum_{i=1}^{\mathrm{n}_{\text {dof }}} c_{i} a_{k}\left(e, N_{i}\right)
$$


and consequently

$$
S^{\star}:=\sum_{k=1}^{\mathrm{n}_{\text {el }}}\left(a_{k}(e, \varphi)-\sum_{i=1}^{\mathrm{n}_{\mathrm{dof}}} c_{i} a_{k}\left(e, N_{i}\right)\right)^{2} .
$$

The normal equations read

$$
\boldsymbol{A}^{\top} \boldsymbol{A} \boldsymbol{c}=\boldsymbol{A}^{\top} \boldsymbol{f}
$$

where $\boldsymbol{A}$ and $\boldsymbol{f}$ are defined componentwise by

$$
A_{i k}=a_{k}\left(e, N_{i}\right) \text { and } f_{k}=a_{k}(e, \varphi) \text { for } i=1, \ldots, \mathrm{n}_{\mathrm{dof}} \text { and } k=1, \ldots, \mathrm{n}_{\mathrm{el}} .
$$

Note that matrix $\boldsymbol{A}^{\top} \boldsymbol{A}$ is usually ill-conditioned and not sparse. Consequently, the system is rather solved directly as the over-determined system

$$
A c \approx f
$$

by using the singular value decomposition of matrix $\boldsymbol{A}$.

Obviously, the obtained value for $v^{H}$ is not optimal but it furnishes a good remedy for error distributions where the phenomenon of alternate signs and error compensation is dramatic.

It is shown in the following example how this strategy is indeed reducing drastically the characteristic values of the local error contributions. Unfortunately, the same example demonstrates that the remeshing criterion associated with this error distribution is not producing meshes with the expected features.

Let us consider the Poisson equation in the square domain $\Omega=]-1,1[\times]-1,1[$ with a source term and (homogeneous) Dirichlet boundary conditions such that the exact solution is

$$
u(x, y):=\exp \left(-10\left(x^{2}+y^{2}\right)\right) \cos (0.5 \pi x) \cos (0.5 \pi y) .
$$

The quantity of interest is defined as the averaged value of the solution in a circle of radius 0.05 centered in the center $P$ of the domain $\Omega$, thus $J(u) \approx$ $u(P)$. The solution of the adjoint problem, $\varphi$, is replaced by a much more accurate approximation, $\varphi^{h}$, computed with an overkill mesh of characteristic size $h=H / 4$.

The approximate solutions are computed in a first step with a uniform mesh of $10 \times 10$ bilinear four-noded quadrilaterals. The error distributions corresponding to $a(e, \varepsilon)$ and $a\left(e, \varphi^{h}-v^{H}\right)$ are shown in Figure 1. Note that computing $v^{H}$ with the methodology introduced in this section reduces significantly the values of the local error components (the scale in the bottom plot is one order 

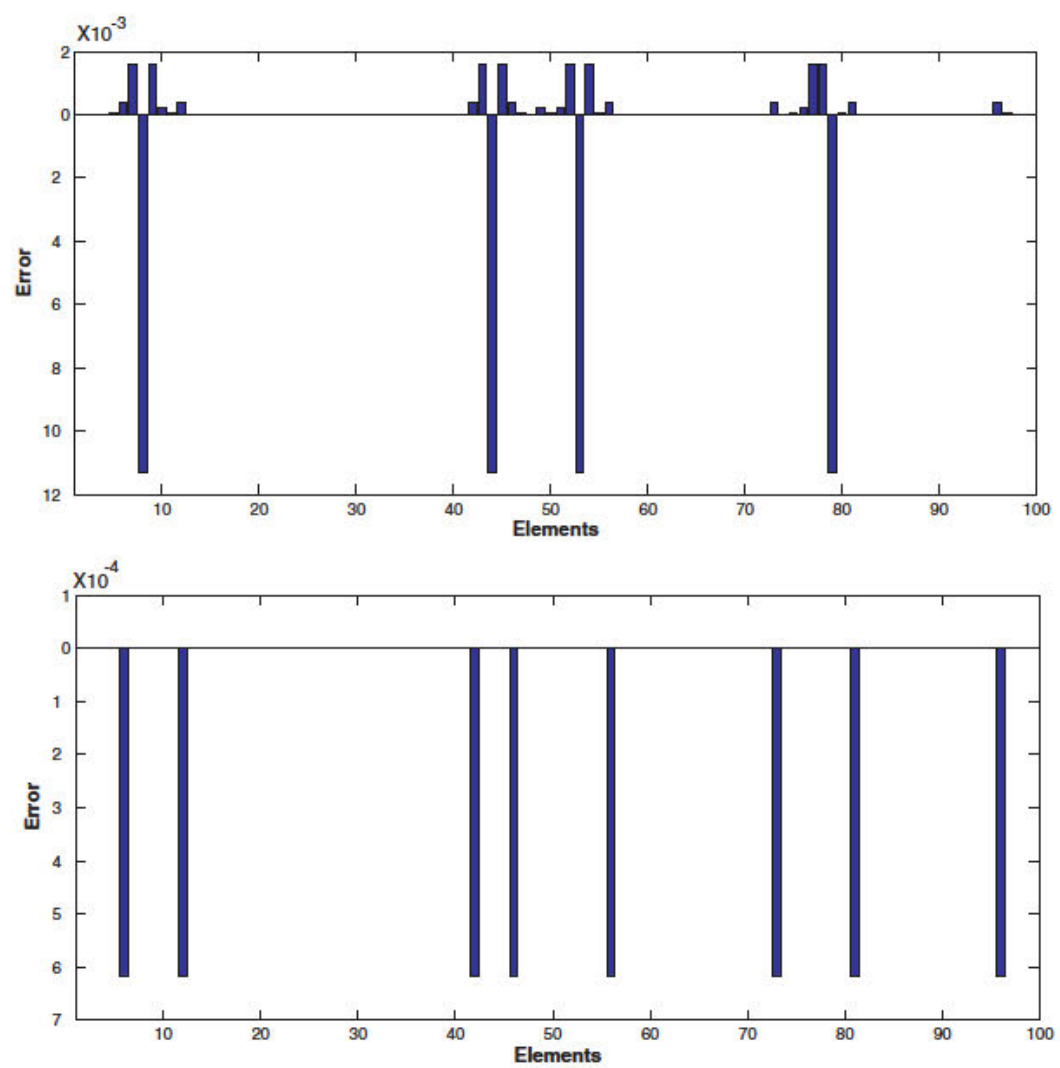

Fig. 1. Element-by-element errors for $a(e, \varepsilon)$ (top) and $a\left(e, \varphi^{h}-v^{H}\right)$ (bottom) for the initial uniform mesh

of magnitude lower). This is quantified by the sum of the absolute values of the local components:

$$
\sum_{k}\left|a_{k}(e, \varepsilon)\right|=6.225 \times 10^{-2} \quad \text { and } \quad \sum_{k}\left|a_{k}\left(e, \varphi^{h}-v^{H}\right)\right|=4.934 \times 10^{-3}
$$

Thus, using $v^{H}$ has the desired effect in the error distribution: minimizes compensation and decreases the local error contributions.

Nevertheless, in practice, using this distribution in the remeshing process is not producing proper meshes. For instance, Figure 2 displays the meshes obtained in the first steps of remeshing processes based on $a(e, \varepsilon)$ (top) and $a\left(e, \varphi^{h}-v^{H}\right)$ (bottom). The error in the uniform starting mesh is of $4.24 \%$ and the target error is $0.05 \%$. Obviously, the meshes generated in the process driven by $a\left(e, \varphi^{h}-v^{H}\right)$ are not acceptable: they are almost uniform. This is probably due to the limitations of the mesh generation tools, that require a distribution of the element size expressed at the nodes, rather than at the elements. Transferring information from elements to nodes smoothes out the sharp variations in the element size required by the remeshing strategy associated with $a\left(e, \varphi^{h}-v^{H}\right)$. This limitation cannot be precluded and therefore using the distribution associated with $a\left(e, \varphi^{h}-v^{H}\right)$ is not realistic for practical 


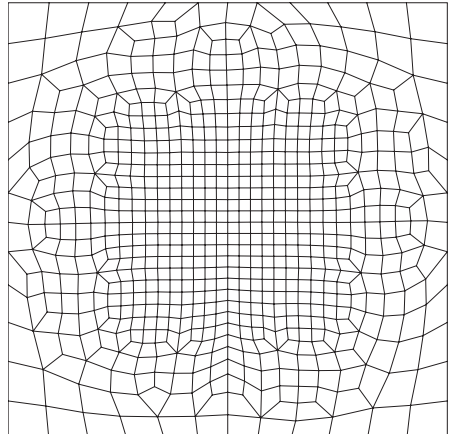

689 el. $\quad 0.23 \%$

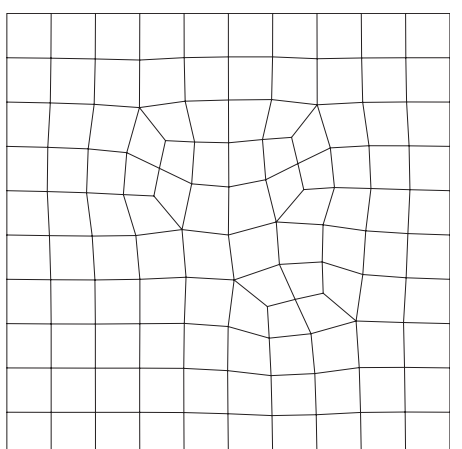

109 el. $\quad 3.5 \%$
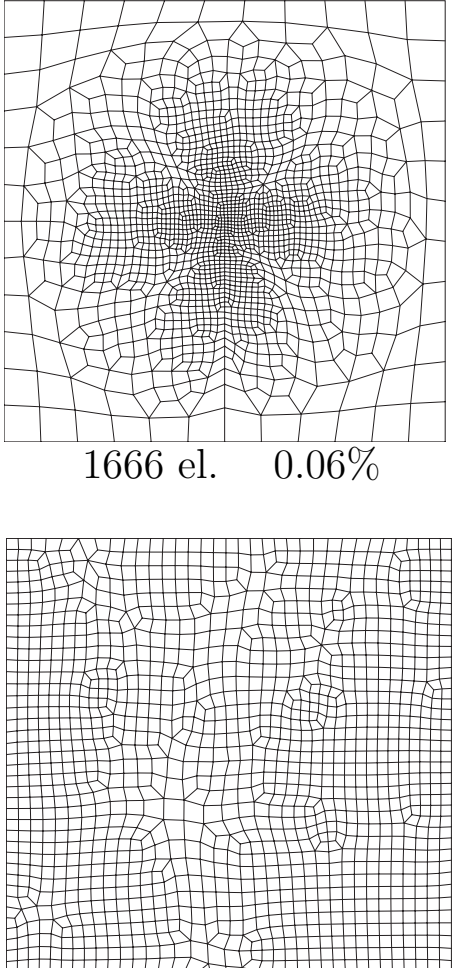

1318 el. $\quad 0.33 \%$

Fig. 2. Meshes in the adaptive process driven by $a(e, \varepsilon)$ (top) and $a\left(e, \varphi^{h}-v^{H}\right)$ (bottom). The number of elements and the error in the quantity of interest are displayed below each mesh

applications.

\subsection{Correcting the alternate signs using a fetch factor}

An alternative and straightforward strategy to obtain a local error representation with the same sign in all the local contributions is to use a proper fetch factor.

Let us introduce the factor

$$
\beta:=\frac{\sum_{k=1}^{\mathrm{n}_{\mathrm{el}}} a_{k}(e, \varepsilon)}{\sum_{k=1}^{\mathrm{n}_{\mathrm{el}}}\left|a_{k}(e, \varepsilon)\right|}=\frac{J(e)}{\sum_{k=1}^{\mathrm{n}_{\mathrm{el}}}\left|a_{k}(e, \varepsilon)\right|} .
$$

Obviously, the error representation

$$
E_{k}=\beta\left|a_{k}(e, \varepsilon)\right|
$$

fulfils (10) and all the contributions have the same sign of $J(e)$. 
Factor $\beta$ is lower than one (in absolute value) and is small if the error distribution $a_{k}(e, \varepsilon)$ has opposite contributions from different parts of the domain, as described in section 5.2.

This correction is expected to work properly if the local rate of convergence of the corrected local error remains constant and can be predicted. That is, if (13) holds and $\alpha$ is known. Numerical evidence demonstrates that the value of $\beta$ does not vary much in the adaptive process and, consequently, the assumption (13) holds also for the corrected error representation. Thus, the resulting remeshing criterion is obviously advantageous with respect to the criterion described in section 5.1 because taking absolute values has no effect in the resulting mesh.

\subsection{Nodal error distribution}

As already mentioned, the output of the remeshing criterion is the input for the mesh generator. Usually, automatic mesh generators require information about the desired mesh size expressed at nodes. Moreover, the element size is described with the simpler interpolation and therefore the information is only needed at the vertices of the elements (nodes of the linear elements). Nevertheless, according to the formulations used above, the output of a remeshing criterion is an element by element mesh size distribution, namely $\widehat{H}_{k}$. Bringing the information to the nodes requires a postprocess involving smoothing of the actual mesh size distribution. Nodal values of the desired element size are computed either averaging the surrounding elements or keeping the minimum element size. Both alternatives introduce deficiencies in the description of the element size distribution, especially where the variations are steep, resulting in a slow convergence and an undesirable oscillating behavior of the adaptive process.

The partition of the unity concept is used to split the error representation involving the weak residual $R^{P}(\cdot)$, see (8) into node by node contributions.

$$
J(e)=R^{P}(\varepsilon)=\sum_{i=1}^{\mathrm{n}_{\mathrm{poin}}} R^{P}\left(\widetilde{N}_{i} \varepsilon\right)
$$

where $\mathrm{n}_{\text {poin }}$ is the number of vertices in the mesh and $\widetilde{N}_{i}, i=1, \ldots, \mathrm{n}_{\text {poin }}$, are the linear scalar shape functions associated with the vertex nodes. Note that $\widetilde{N}_{i}$ and $N_{i}$ coincide for scalar problems and linear elements but they are formally different either if the numbers of unknowns per node is larger than one (as, for instance, in the mechanical 2D or 3D setting) or if the elements are of higher degree. 
Thus, taking $E_{k}=R^{P}\left(\widetilde{N}_{k} \varepsilon\right), k=1, \ldots, \mathrm{n}_{\text {poin }}$, yields a nodal local error representation that is used to derive new remeshing criteria. Note that with this definition of $E_{k},(10)$ holds if $\mathrm{n}_{\mathrm{e} 1}$ is replaced by $\mathrm{n}_{\text {poin }}$. Two further assumptions are required:

(1) A nodal element size is defined using some averaging. For instance, the element size corresponding to node $i$ is taking as the measure of the support of $\widetilde{N}_{i}$ to the power of $1 / d$, eventually divided by two.

(2) The nodal contribution to the error converges locally at a given rate $\alpha$, exactly as indicated in (13) but with both $H_{k}$ and $E_{k}$ defined nodally.

Note that, for the sake of a simple presentation, the notations for $E_{k}$ and $H_{k}$ are kept the same as for the element by element approach.

If these assumptions are fulfilled, the remeshing criteria are derived exactly in the same way as before, just replacing $\mathrm{n}_{\mathrm{el}}$ by $\mathrm{n}_{\mathrm{poin}}$ all along the process. Obviously, when following this approach the criterion analyzed in section 4 is optimal in the sense that it minimizes the resulting number of nodes, not the number of elements.

An alternative approach is based on using the weak residual of the adjoint problem. The error in the quantity of interest is also represented by

$$
J(e)=R^{P}(e)=\sum_{i=1}^{\mathrm{n}_{\text {poin }}} R^{D}\left(\widetilde{N}_{i} e\right),
$$

where $R^{D}(\cdot):=J(\cdot)-a\left(\cdot, \varphi^{H}\right)$ is the weak residual of the adjoint problem. Taking $E_{k}=R^{D}\left(\widetilde{N}_{k} e\right)$, the same rationale is used to find the corresponding remeshing criteria, with exactly the same expressions as before.

\section{NUMERICAL EXAMPLES}

The remeshing criteria introduced in the previous sections are tested by solving adaptively both a thermal problem with a synthetic analytical solution and a classical mechanical test in linear elasticity. The mechanical test uses the geometry and the loading setup of the so-called Single Edge Notched Beam (SENB) mostly used in fracture mechanics [12]. The automatic mesh generator developed in [13] is used to generate the meshes along the remeshing procedure. It is worth noting that no error estimation is used in the numerical experiments. Recall that the goal is to test the remeshing criteria. Thus, in the cases where the exact solution is not available, in order to avoid introducing a new source of uncertainty, the error is not estimated but computed by using an 
overkill mesh of element size $h=H / 4$, i.e. by uniformly splitting each element of the current mesh into 16 subelements.

\subsection{Scalar problem (Poisson equation)}

The Poisson equation is solved in the squared domain $\Omega=] 0,1[\times] 0,1[$. The source term and Dirichlet boundary conditions are taken such that the exact solution is

$$
u(x, y)=x(1-x) y\left(1-y^{2}\right)\left(1+200 x^{2}+7 y\right)
$$

The quantity of interest is the averaged value of $u$ in the circle of center $P=(0.9,0.9)$ and radius 0.05 .

The adaptive procedure starts with a uniform mesh of $8 \times 8$ elements. The relative error in the quantity of interest in the first mesh is $7 \%$. The target error in the adaptive process is $0.05 \%$ which is equivalent to obtain 3 correct significant digits in the approximation of the quantity of interest [14].

\begin{tabular}{|c|rrrr|rr|rr|rrrrr|}
\hline \multirow{2}{*}{ mesh } & \multicolumn{2}{|c|}{$a(e, \varepsilon)$} & \multicolumn{2}{|c|}{$\beta$} & \multicolumn{2}{|c|}{$R^{P}(e)$} & \multicolumn{2}{c|}{$R^{D}(\varepsilon)$} & \multicolumn{3}{c|}{$\beta$} \\
& \# el & error & \# el & error & \# el & error & \# el & error & \# el & error & \# el & error \\
\hline 0 & 64 & $7.0 \%$ & & & & & & & & & & \\
1 & 557 & $0.47 \%$ & 506 & $0.56 \%$ & 613 & $0.55 \%$ & 571 & $0.54 \%$ & 592 & $0.51 \%$ & 586 & $0.53 \%$ \\
2 & 1535 & $0.09 \%$ & 1284 & $0.11 \%$ & 1553 & $0.13 \%$ & 1403 & $0.11 \%$ & 1574 & $0.09 \%$ & 1473 & $0.09 \%$ \\
3 & 2333 & $0.04 \%$ & 887 & $0.12 \%$ & 2464 & $0.04 \%$ & 1924 & $0.05 \%$ & 2469 & $0.04 \%$ & 1811 & $0.06 \%$ \\
4 & 2461 & $0.04 \%$ & 926 & $0.14 \%$ & 2398 & $0.04 \%$ & 1688 & $0.07 \%$ & 2195 & $0.05 \%$ & 1721 & $0.05 \%$ \\
5 & 2474 & $0.04 \%$ & 1030 & $0.11 \%$ & 2327 & $0.04 \%$ & 1923 & $0.05 \%$ & 2595 & $0.04 \%$ & 1611 & $0.07 \%$ \\
\hline
\end{tabular}

Thermal problem: results provided by different strategies based on the UED criterion

\begin{tabular}{|c|rrrr|rr|rr|rrrrr|}
\hline \multirow{2}{*}{ mesh } & \multicolumn{2}{|c}{$a(e, \varepsilon)$} & \multicolumn{2}{|c}{$\beta$} & \multicolumn{2}{c|}{$R^{P}(e)$} & \multicolumn{2}{c|}{$\beta$} & \multicolumn{2}{c|}{$R^{D}(\varepsilon)$} & \multicolumn{3}{c|}{$\beta$} \\
& \# el & error & \# el & error & \# el & error & \# el & error & \# el & error & \# el & error \\
\hline 0 & 64 & $7.0 \%$ & 64 & $7.0 \%$ & 64 & $7.0 \%$ & 64 & $7.0 \%$ & 64 & $7.0 \%$ & 64 & $7.0 \%$ \\
1 & 381 & $0.55 \%$ & 369 & $0.55 \%$ & 423 & $0.55 \%$ & 429 & $0.55 \%$ & 379 & $0.62 \%$ & 380 & $0.62 \%$ \\
2 & 2367 & $0.06 \%$ & 2076 & $0.07 \%$ & 2309 & $0.07 \%$ & 2157 & $0.07 \%$ & 2381 & $0.09 \%$ & 2160 & $0.11 \%$ \\
3 & 7645 & $0.02 \%$ & 3124 & $0.09 \%$ & 8163 & $0.03 \%$ & 6549 & $0.04 \%$ & 7740 & $0.02 \%$ & 6613 & $0.03 \%$ \\
\hline
\end{tabular}

Thermal problem: results provided by different strategies based on the USE criterion

The UED and USE remeshing criteria are used for the error representations corresponding to $E_{k}=\left|a_{k}(e, \varepsilon)\right|, E_{k}=\left|R^{P}\left(\widetilde{N}_{k} \varepsilon\right)\right|, E_{k}=\left|R^{D}\left(\widetilde{N}_{k} e\right)\right|$ and the corrected distribution using the proper fetch factors $\beta$. Thus, 6 sequences of meshes are obtained for each one of the two remeshing criteria. The number of remeshing steps in each process is set to 5 for the UED criterion and 3 


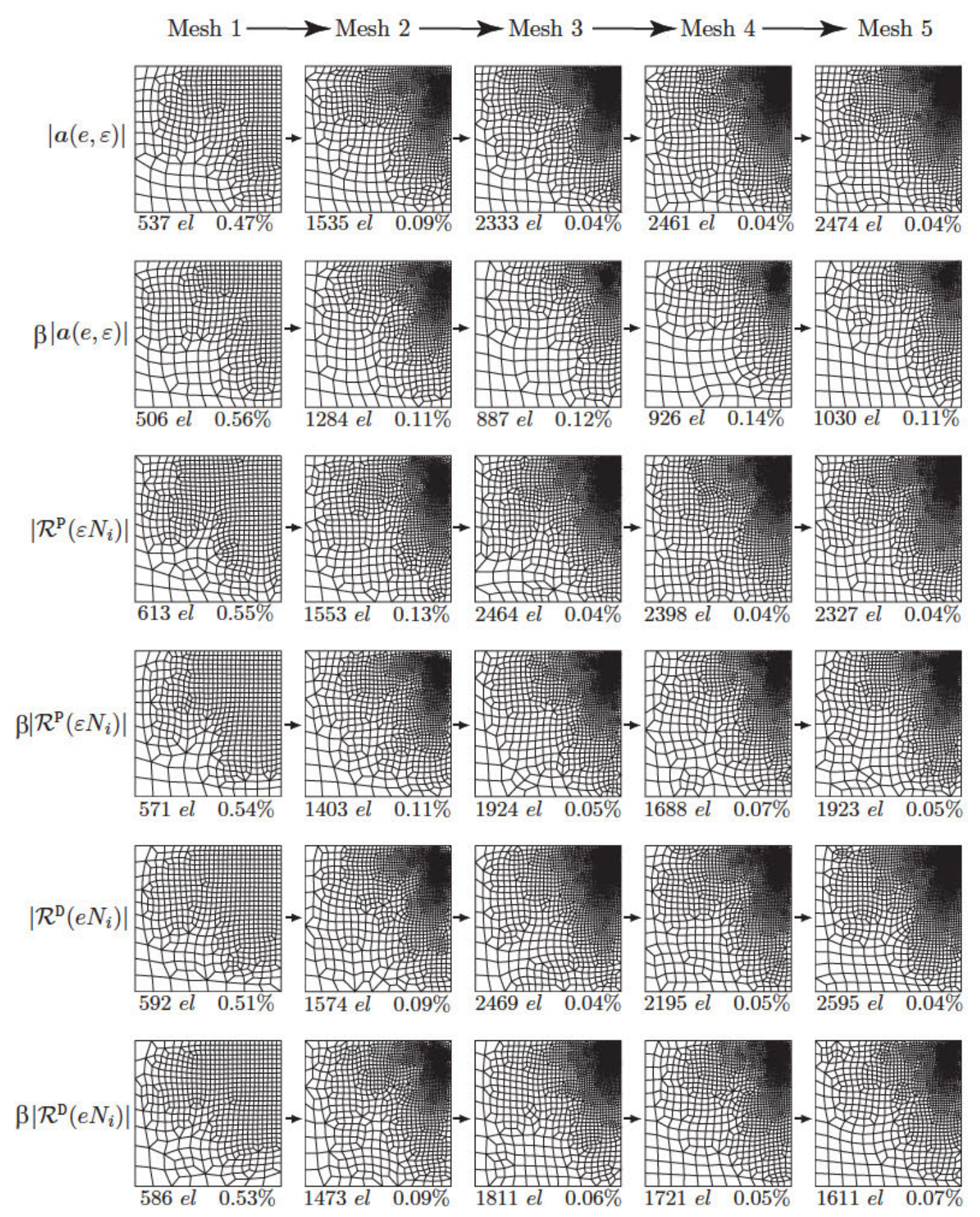

Fig. 3. Thermal problem: meshes obtained with UED remeshing criterion

for the USE criterion. The adaptive process is not stopped even if the target accuracy is reached before. This is done in order to test the consistency of the remeshing strategies.

A summary of the results obtained for the thermal problem are displayed in tables 1 and 2 . In this example, the convergence behavior of all the adapted strategies is more efficient than the uniform refinement. The observed converge rate almost equal for all the adaptive procedures and, naturally, larger than 


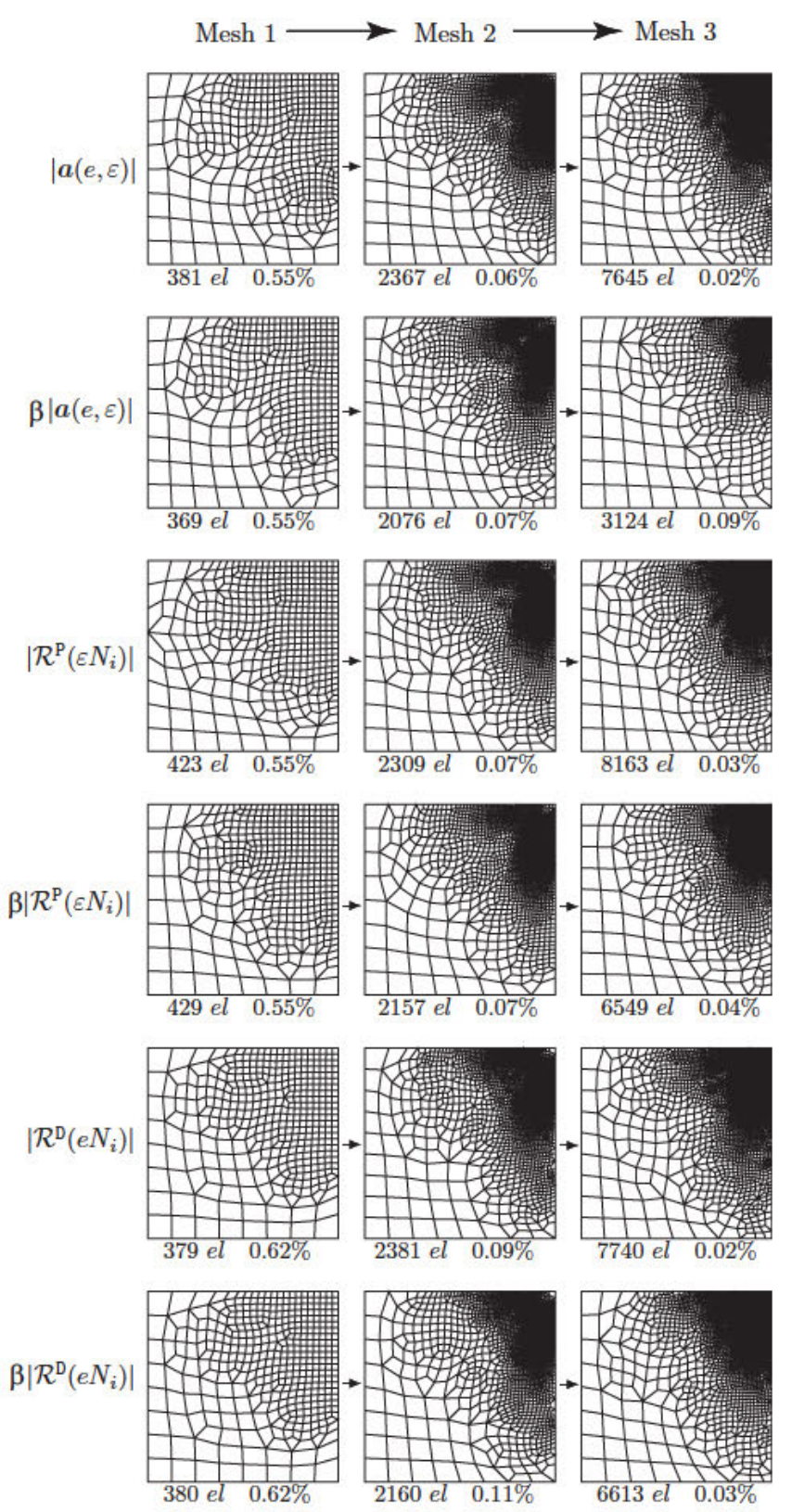

Fig. 4. Thermal problem: meshes obtained with USE remeshing criterion

for the uniform refinement. The meshes obtained are plotted in figures 3 and 4.

It can be concluded that UED criterion yields meshes with much fewer elements than the USE criterion, as expected. Moreover, for the UED criterion the uncorrected error representation corresponding to $E_{k}=\left|a_{k}(e, \varepsilon)\right|$ is too conservative in the sense that it produces meshes with more elements than needed. The use of the $\beta$ factor reduces the number of required elements. Nevertheless, in the case where $E_{k}=\left|a_{k}(e, \varepsilon)\right|$, the error error remains larger than the prescribed target. This is due to the smoothing in the element size 

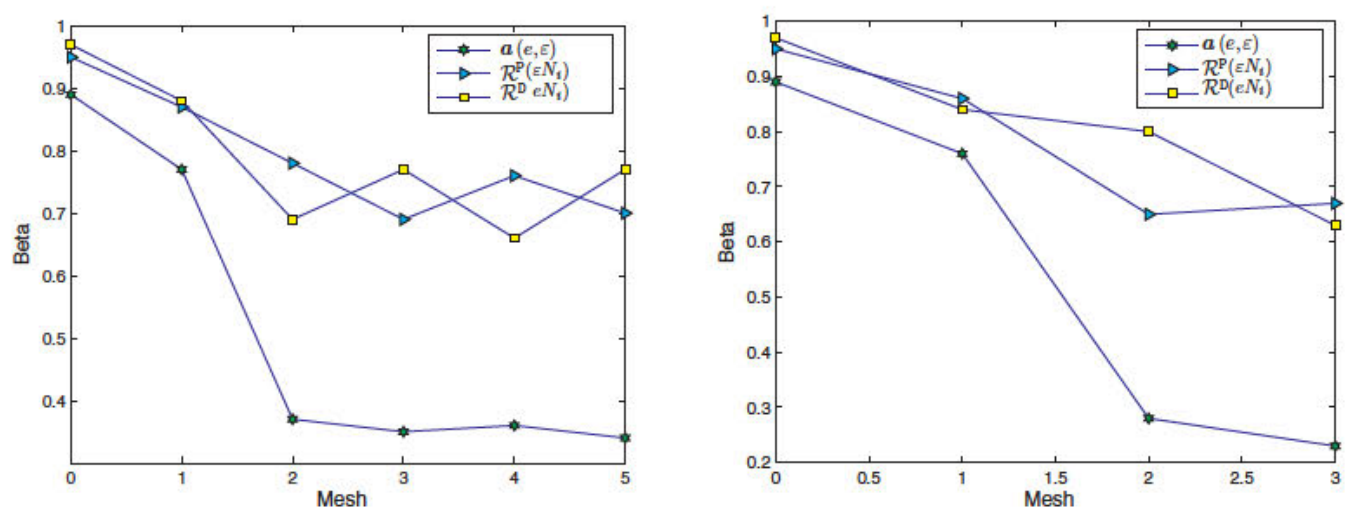

Fig. 5. Thermal problem: evolution of the values of $\beta$ along the remeshing process for the strategies using the $\beta$ factor and both the UED (left) and the USE (right) criteria.

distribution introduced by the transfer from elements to nodes required by the mesh generator. In fact, the strategies based on the nodal descriptions of the error $E_{k}=\left|R^{P}\left(\widetilde{N}_{k} \varepsilon\right)\right|$ and $E_{k}=\left|R^{D}\left(\widetilde{N}_{k} e\right)\right|$ and corrected with factor $\beta$ are in general much sharper and reach an accuracy very close to the prescribed target $(0.05 \%)$ with meshes with fewer elements.

The evolution of factor $\beta$ along the remeshing processes is shown in figure 5 . It is worth noting that the values of $\beta$ are practically constant, once the configuration of the mesh is close to the optimal one.

\subsection{Single Edge Notched Beam (SENB)}

The problem setup described in figure 6 corresponds to the classical SENB test, introduced in [12] and extensively used to assess the fractural behavior of concrete and other brittle materials. Here, the same geometry and loads are used with a linear elastic model (with Poisson coefficient $\nu=0.33$ ).

\begin{tabular}{|c|cccc|cc|cc|ccc|cc|}
\hline \multirow{2}{*}{ mesh } & \multicolumn{2}{|c}{$a(e, \varepsilon)$} & \multicolumn{2}{c|}{$\beta$} & \multicolumn{2}{c|}{$R^{P}(\varepsilon)$} & \multicolumn{2}{c|}{$\beta$} & \multicolumn{2}{c|}{$R^{D}(e)$} & \multicolumn{3}{c|}{$\beta$} \\
& \# el & error & \# el & error & \# el & \# el & \# el & error & \# el & error & \# el & error \\
\hline 0 & 414 & $10.7 \%$ & 414 & $10.7 \%$ & 414 & $10.7 \%$ & 414 & $10.7 \%$ & 414 & $10.7 \%$ & 414 & $10.7 \%$ \\
1 & 554 & $3.35 \%$ & 369 & $3.61 \%$ & 579 & $1.60 \%$ & 363 & $7.17 \%$ & 530 & $5.52 \%$ & 338 & $6.41 \%$ \\
2 & 625 & $1.45 \%$ & 280 & $12.7 \%$ & 711 & $1.35 \%$ & 291 & $5.68 \%$ & 692 & $0.58 \%$ & 406 & $11.5 \%$ \\
3 & 687 & $1.06 \%$ & 313 & $8.02 \%$ & 719 & $0.02 \%$ & 273 & $6.85 \%$ & 689 & $1.14 \%$ & 240 & $8.33 \%$ \\
\hline
\end{tabular}

Table 3

Mechanical problem with CMOD quantity of interest: results provided by different strategies based on the UED criterion

The quantities of interest in this test are both the horizontal and vertical separations of the notch. These are denoted by Crack Mouth Opening Displacement (CMOD) and Crack Mouth Sliding Displacement (CMSD) respectively. 


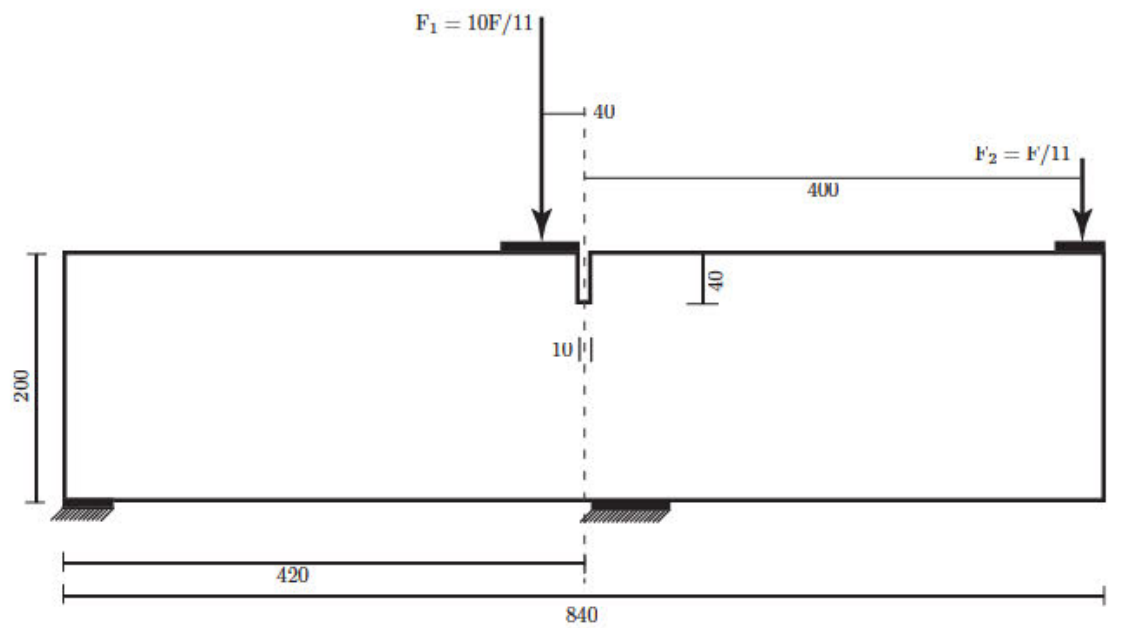

Fig. 6. Mechanical problem: problem statement

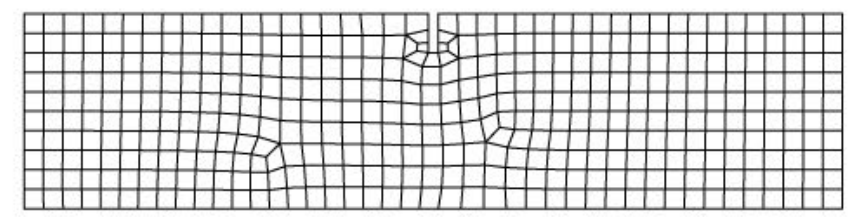

Fig. 7. Mechanical problem: initial mesh (414 elements).

\begin{tabular}{|c|rc|rc|rr|rr|rrrrr|}
\hline \multirow{2}{*}{ mesh } & \multicolumn{2}{|c}{$a(e, \varepsilon)$} & \multicolumn{2}{c|}{$\beta$} & \multicolumn{2}{c|}{$R^{P}(\varepsilon)$} & \multicolumn{2}{c|}{$\beta$} & \multicolumn{2}{c|}{$R^{D}(e)$} & \multicolumn{3}{c|}{$\beta$} \\
& \# el & error & \# el & error & \# el & error & \# el & error & \# el & error & \# el & error \\
\hline 0 & 414 & $10.7 \%$ & 414 & $10.7 \%$ & 414 & $10.7 \%$ & 414 & $10.7 \%$ & 414 & $10.7 \%$ & 414 & $10.7 \%$ \\
1 & 858 & $1.93 \%$ & 565 & $2.91 \%$ & 937 & $2.14 \%$ & 550 & $2.94 \%$ & 858 & $4.12 \%$ & 762 & $7.4 \%$ \\
2 & 2375 & $0.85 \%$ & 710 & $1.52 \%$ & 2969 & $0.78 \%$ & 821 & $2.77 \%$ & 2349 & $1.03 \%$ & 1901 & $0.74 \%$ \\
3 & 5794 & $0.64 \%$ & 922 & $0.47 \%$ & 6836 & $0.30 \%$ & 2104 & $1.48 \%$ & 5668 & $0.49 \%$ & 2690 & $0.06 \%$ \\
\hline
\end{tabular}

Table 4

Mechanical problem with CMOD quantity of interest: results provided by different strategies based on the USE criterion

\begin{tabular}{|c|rrrr|rr|rr|rrrr|r|}
\hline \multirow{2}{*}{ mesh } & \multicolumn{2}{|c}{$a(e, \varepsilon)$} & \multicolumn{2}{c}{$\beta$} & \multicolumn{2}{c|}{$R^{P}(\varepsilon)$} & \multicolumn{2}{c|}{$\beta$} & \multicolumn{2}{c|}{$R^{D}(e)$} & \multicolumn{3}{c|}{$\beta$} \\
& \# el & error & \# el & error & \# el & error & \# el & error & \# el & error & \# el & error \\
\hline 0 & 414 & $9.45 \%$ & 414 & $9.45 \%$ & 414 & $9.45 \%$ & 414 & $9.45 \%$ & 414 & $9.45 \%$ & 414 & $9.45 \%$ \\
1 & 860 & $2.89 \%$ & 839 & $2.84 \%$ & 923 & $2.44 \%$ & 802 & $2.57 \%$ & 887 & $2.48 \%$ & 780 & $2.35 \%$ \\
2 & 1443 & $0.83 \%$ & 1112 & $0.91 \%$ & 1312 & $0.89 \%$ & 1159 & $0.99 \%$ & 1248 & $1.08 \%$ & 1106 & $1.0 \%$ \\
3 & 1622 & $0.54 \%$ & 912 & $1.01 \%$ & 1473 & $0.58 \%$ & 1220 & $0.73 \%$ & 1477 & $0.59 \%$ & 1157 & $0.77 \%$ \\
4 & 1577 & $0.48 \%$ & 868 & $1.02 \%$ & 1629 & $0.43 \%$ & 1196 & $0.66 \%$ & 1609 & $0.45 \%$ & 1237 & $0.6 \%$ \\
\hline
\end{tabular}

Table 5

Mechanical problem with CMSD quantity of interest: results provided by different strategies based on the UED criterion

All the adaptive procedures start from the same initial mesh shown in figure 7. A uniform remeshing procedure is also performed in order to highlight the 

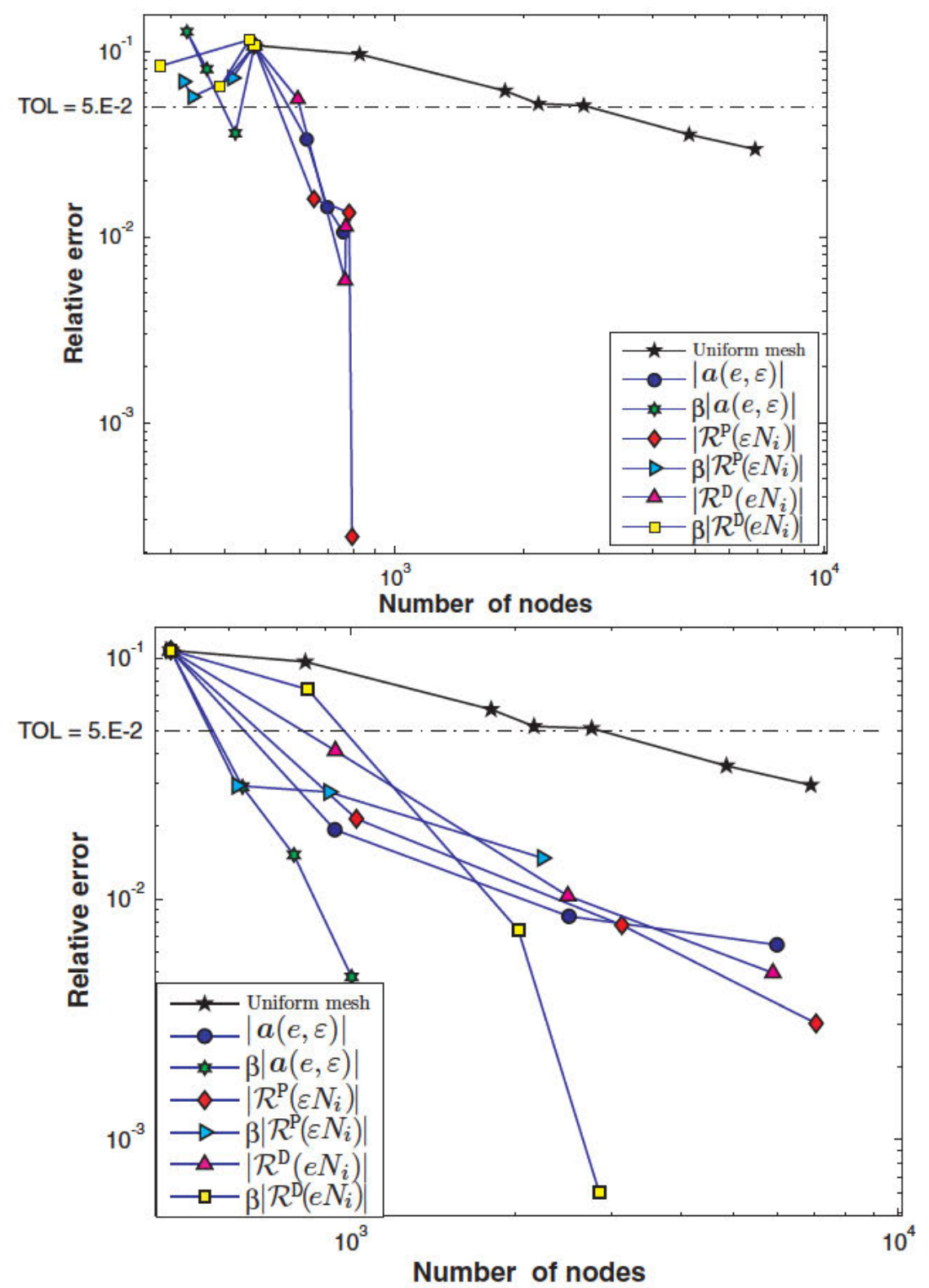

Fig. 8. Mechanical problem with CMOD quantity of interest: convergence curves for the different strategies using the UED criterion (top) and the USE criterion (bottom). The curve marked with $\star$ corresponds to a uniform remeshing.

advantages of the adaptive strategies. The error in the first mesh is $10.7 \%$ for the CMOD quantity and $9.45 \%$ for CMSD. The target error is set to $5 \%$ for the CMOD quantity of interest and to $0.5 \%$ for the CMSD. Note that these error prescriptions are equivalent to require one and two correct significant digits respectively for CMOD and CMSD, see [14].

The results are described using the same structure as in the previous example. A summary of the results obtained for the mechanical problem with the 


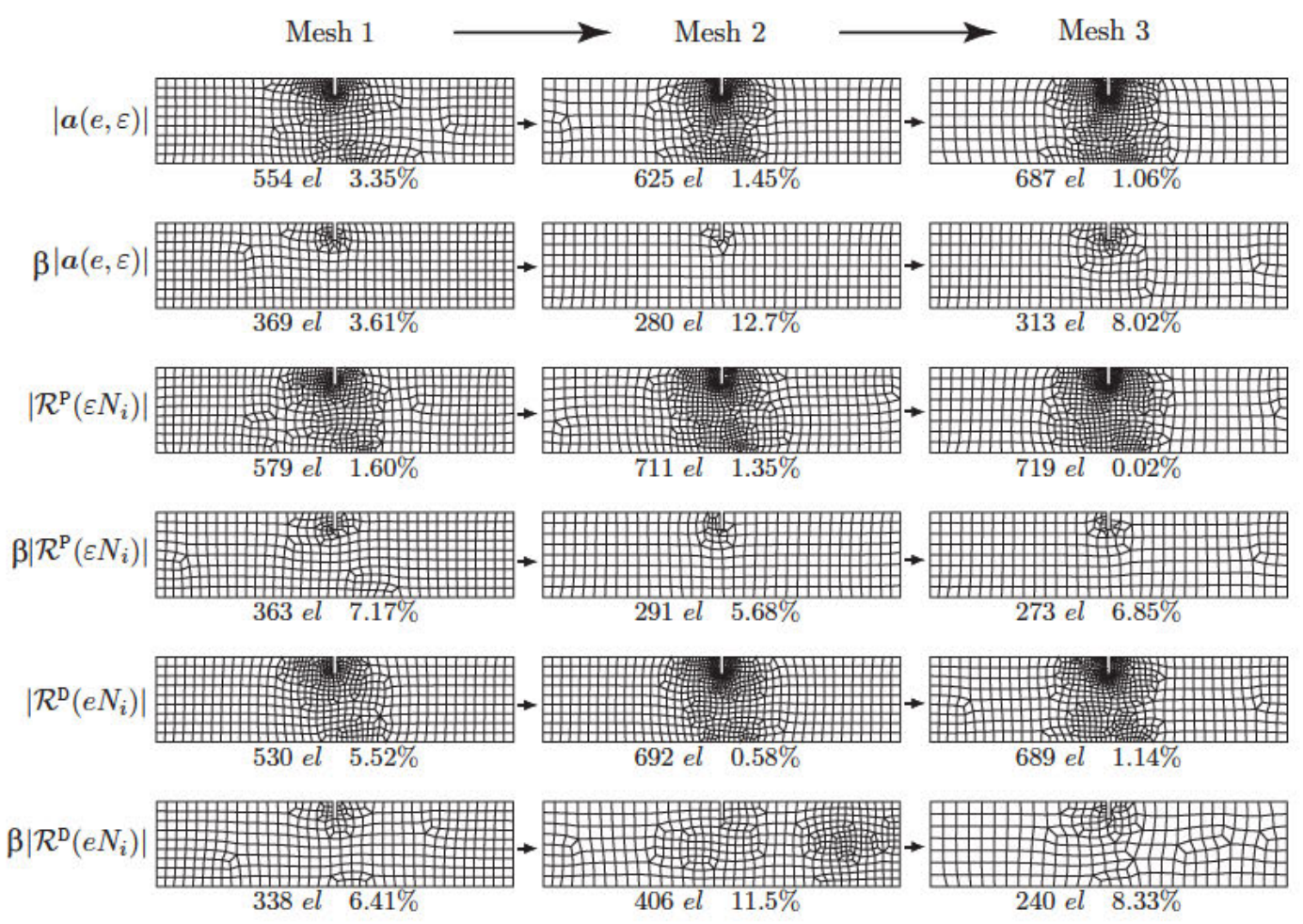

Fig. 9. Mechanical problem with CMOD quantity of interest: meshes obtained with UED remeshing criterion

\begin{tabular}{|c|c|c|c|c|c|c|c|c|c|c|c|c|}
\hline \multirow{2}{*}{ mesh } & \multicolumn{2}{|c|}{$a(e, \varepsilon)$} & \multicolumn{2}{|c|}{$\beta$} & \multicolumn{2}{|c|}{$R^{P}(\varepsilon)$} & \multicolumn{2}{|c|}{$\beta$} & \multicolumn{2}{|c|}{$R^{D}(e)$} & \multicolumn{2}{|c|}{$\beta$} \\
\hline & $\#$ el & error & \# el & error & $\#$ el & error & \# el & error & $\#$ el & error & $\#$ el & error \\
\hline 0 & 414 & $9.45 \%$ & 414 & $9.45 \%$ & 414 & $9.45 \%$ & 414 & $9.45 \%$ & 414 & $9.45 \%$ & 414 & $9.45 \%$ \\
\hline 1 & 1063 & $2.28 \%$ & 1030 & $2.25 \%$ & 1109 & $2.31 \%$ & 1039 & $2.17 \%$ & 1119 & $2.19 \%$ & 976 & $2.30 \%$ \\
\hline 2 & 4248 & $0.52 \%$ & 3349 & $0.53 \%$ & 4275 & $0.49 \%$ & 3986 & $0.50 \%$ & 4418 & $0.47 \%$ & 4568 & $0.49 \%$ \\
\hline 3 & 13242 & $0.16 \%$ & 7142 & $0.23 \%$ & 12838 & $0.15 \%$ & 11811 & $0.15 \%$ & 13131 & $0.16 \%$ & 12758 & $0.14 \%$ \\
\hline
\end{tabular}

Table 6

Mechanical problem with CMSD quantity of interest: results provided by different strategies based on the USE criterion

CMOD quantity of interest are displayed in tables 3 and 4. Figure 8 shows the convergence curves obtained along the adaptive processes, compared also with a uniform refinement. The meshes obtained are plotted in figures 9 and 10. The results corresponding to the CMSD quantity of interest are shown in tables 5 and 6 and figures 11, 12 and 13.

The conclusions are for this case quite similar to the previous one. The USE criterion produces meshes with a number of elements much larger than the UED criterion. Moreover, once the proper mesh configuration is attained, the UED criterion keeps the number of elements almost uniform. On the contrary, the USE criterion refines the meshes further on, even if the prescribed accuracy is already reached. Thus, the conclusion is that the UED criterion is consistent 


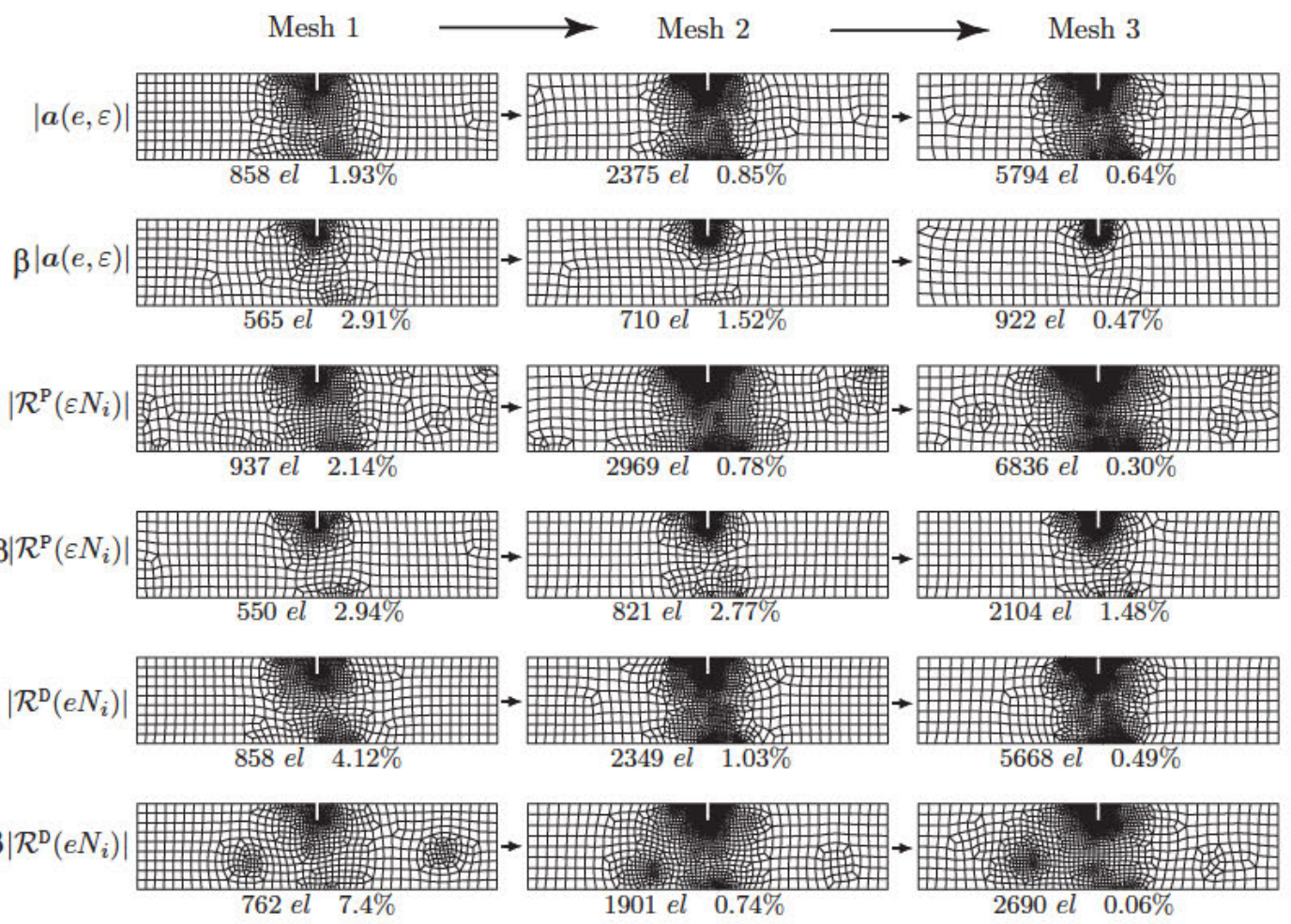

Fig. 10. Mechanical problem with CMOD quantity of interest: meshes obtained with USE remeshing criterion

and leads to stable adaptive procedures, contrary to the USE criterion.

In the results presented in this section the $\beta$ factor combined with the different versions of the UED criterion provides meshes with an error slightly larger than the prescribed tolerance. This is due to the sharpness of the corresponding remeshing criteria. In other words, the criterion produces the mesh size distribution strictly necessary to obtain the tolerance and therefore any variation in the element size distribution introduced by the mesh generator precludes reaching the tolerance.

\section{Concluding remarks}

A general approach to remeshing criteria for goal oriented $h$-adaptivity has been presented. The UED (Uniform Error Distribution) criterion has been proved to be optimal in the sense that it produces meshes with the least number of elements. The numerical tests confirm this theoretical result.

The desired element size in the new mesh is calculated using the absolute value of the error because the local error contributions have, in general, different signs. A fetch factor, $\beta$, is introduced in order to balance the conservative 

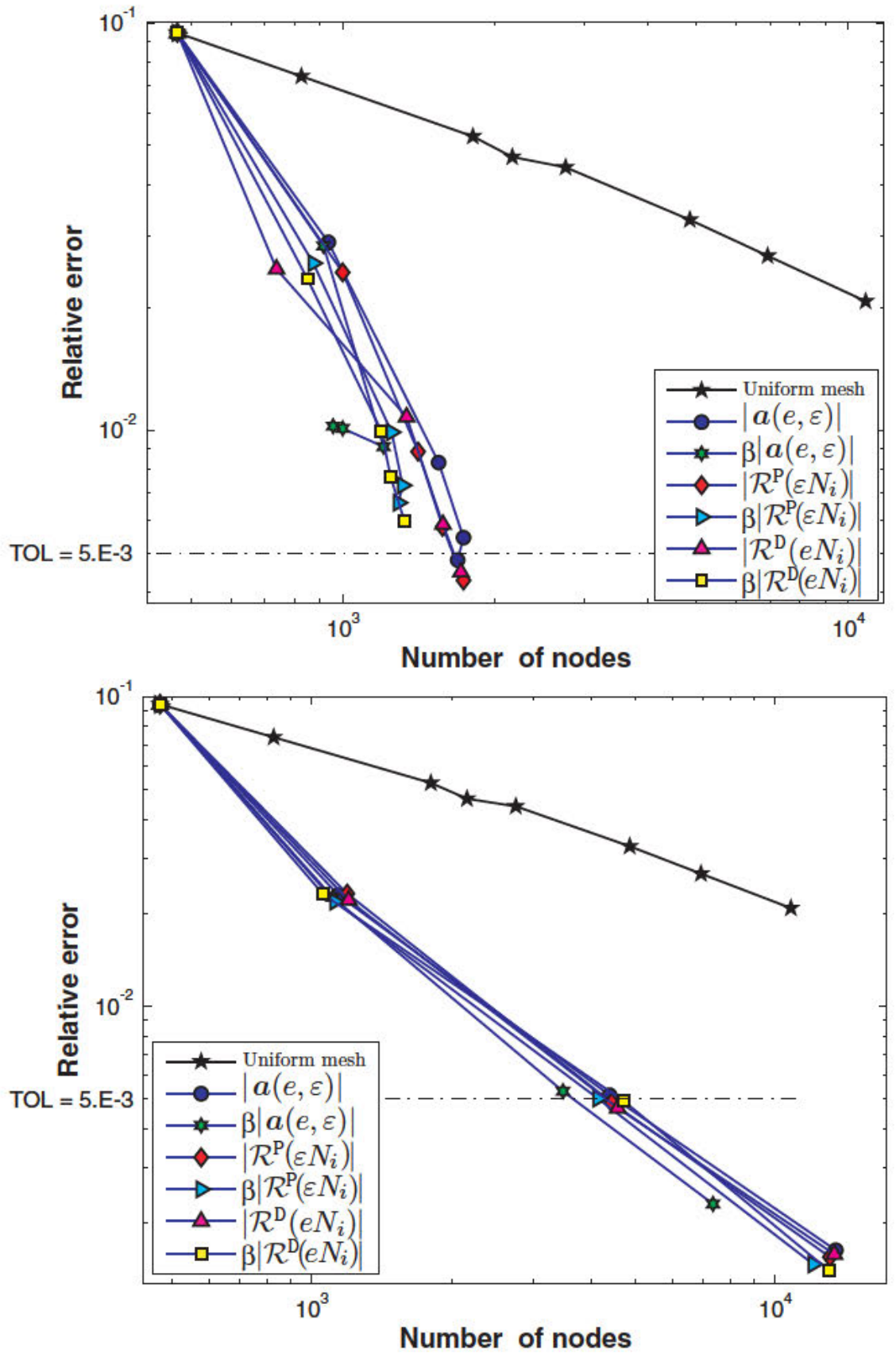

Fig. 11. Mechanical problem with CMSD quantity of interest: convergence curves for the different strategies using the UED criterion (top) and the USE criterion (bottom). The curve marked with $\star$ corresponds to a uniform remeshing.

effect of taking absolute values and reducing the number of elements in the new mesh. The remeshing strategies using factor $\beta$ are very efficient and produce the meshes with the least number of elements.

The transfer of information from the error estimate and the mesh generator is improved by expressing directly in the nodes the output of the remeshing strategy, instead of giving values element by element. 


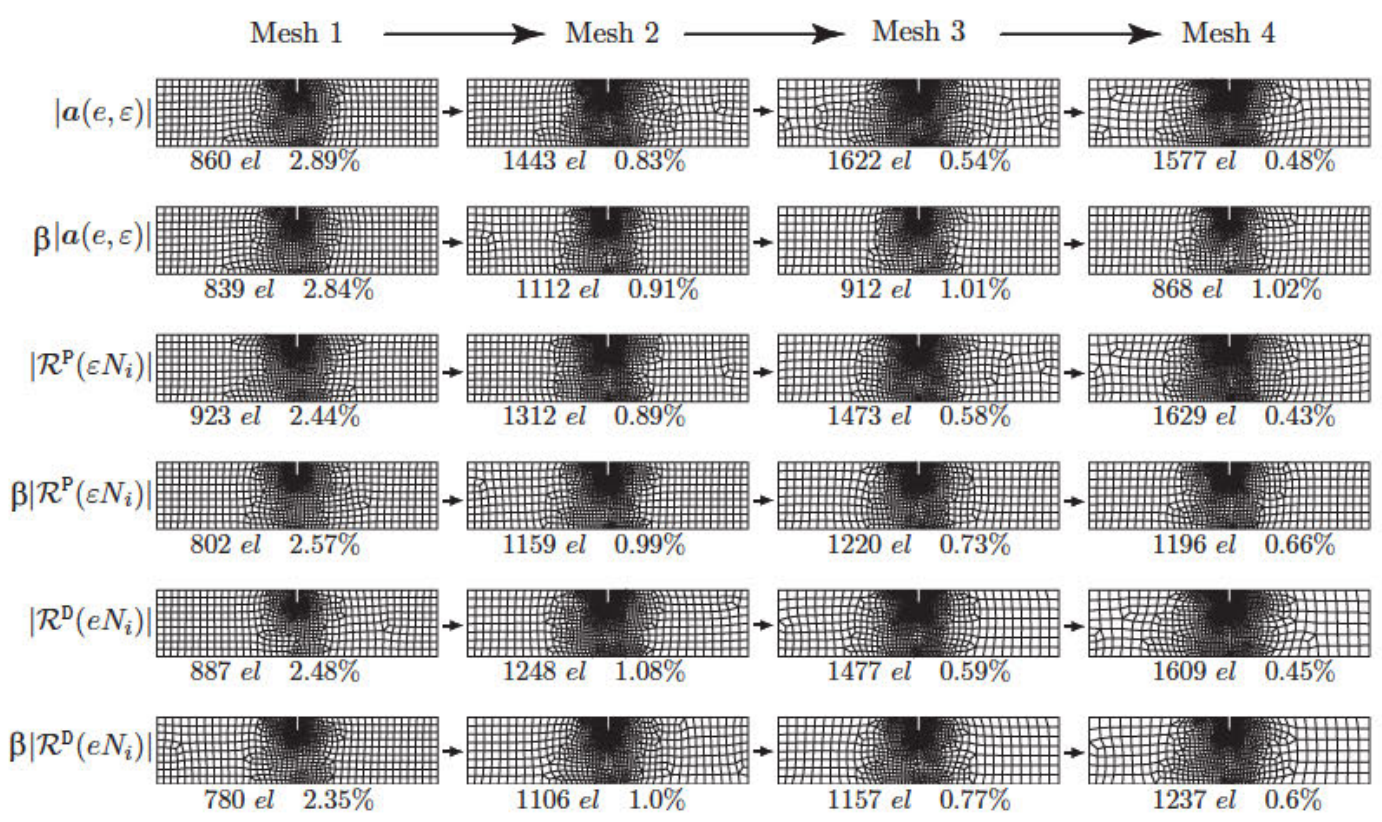

Fig. 12. Mechanical problem with CMSD quantity of interest: meshes obtained with UED remeshing criterion

Mesh 1

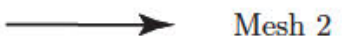

Mesh 3
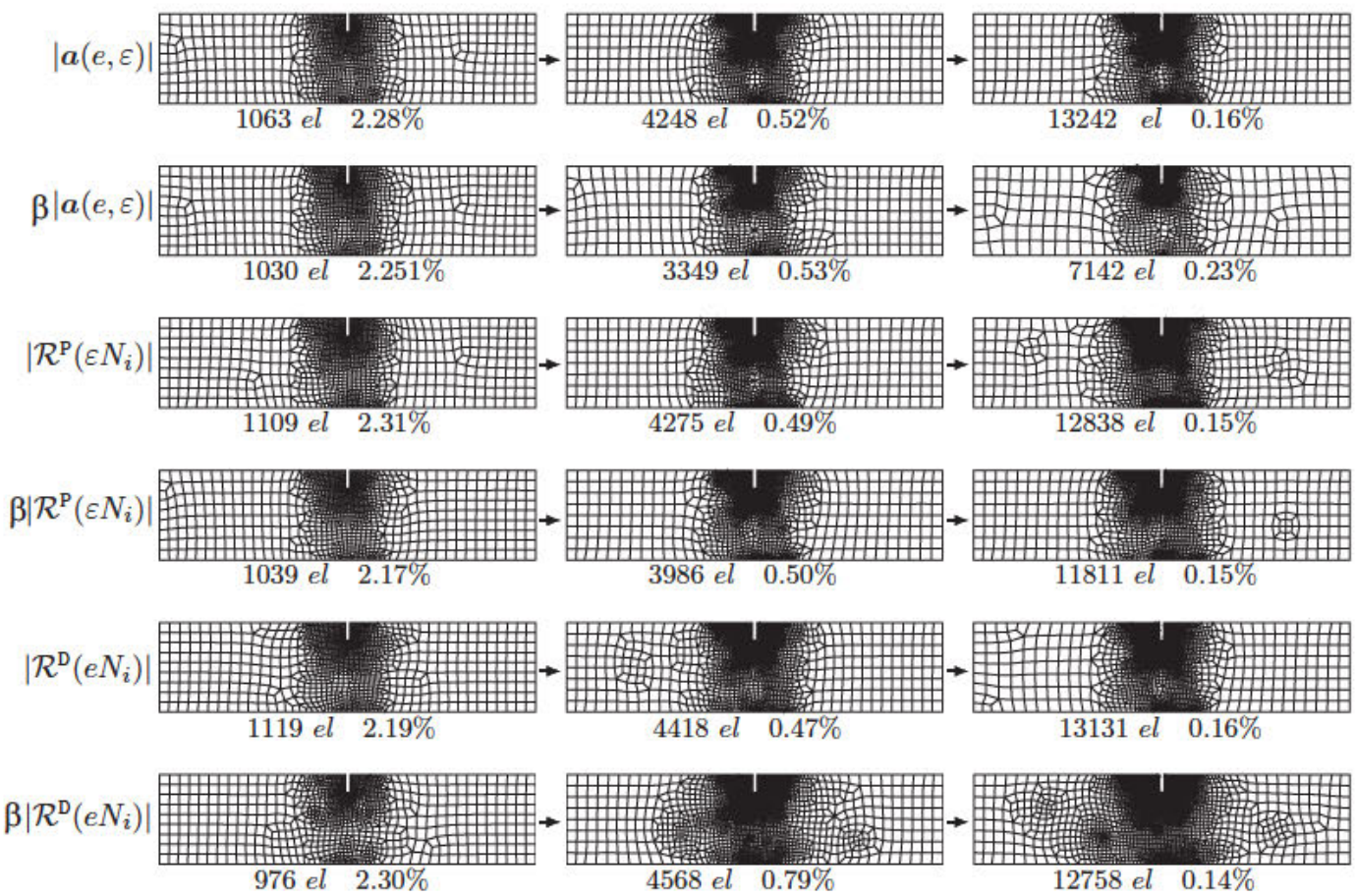

Fig. 13. Mechanical problem with CMSD quantity of interest: meshes obtained with USE remeshing criterion

The analysis of the numerical examples shows that the optimal strategy is to use factor $\beta$ combined with one of the nodal error representations. Moreover, due to the sharpness of the resulting criteria, this has to be used reducing the 
target error to eliminate the effect of the discrepancy between the desired and the obtained element size distribution.

\section{References}

[1] L. Li, P. Bettess, J. Bull, T. Bond, I. Applegarth, Theoretical formulations for adaptive finite element formulations, Comm. Numer. Methods Engrg. 11 (1995) 239-249.

[2] L. Li, P. Bettess, Notes on mesh optimal criteria in adaptive finite element computations, Comm. Numer. Methods Engrg. 11 (1995) 911-915.

[3] E. Oñate, G. Bugeda, A study of mesh optimality criteria in adaptive finite element analysis, Engineering Computations 10 (1993) 307-321.

[4] P. Díez, A. Huerta, A unified approach to remeshing strategies for finite element h-adaptivity, Computer Methods in Applied Mechanics and Engineering 176 (1999) 215-229.

[5] S. Prudhomme, J. Oden, On goal-oriented error estimation for elliptic problems: Application to the control of pointwise errors, Computer Methods in Applied Mechanics and Engineering 176 (1999) 313-331.

[6] J. Peraire, Computing bounds for functional outputs of exact solutions of partial differential equations, in: N. Wiberg, P. Díez (Eds.), Adaptive Modeling and Simulation, Vol. 1, CIMNE, 2003, pp. 4-5.

[7] N. Parés, J. Bonet, A. Huerta, J. Peraire, Guaranteed bounds for linear outputs of interest in elasticity, in: N. Wiberg, P. Díez (Eds.), Adaptive Modeling and Simulation, Vol. 1, CIMNE, 2003, pp. 78-79.

[8] W. Dorfler, A convergent adaptive algorithm for poisson's equation, SIAM Journal on Numerical Analysis 33 (3) (1996) 1106-1124.

[9] P. Morin, R. Nochetto, K. Siebert, Data oscillation and convergence of adaptive fem, SIAM Journal on Numerical Analysis 38 (2) (2000) 466-488.

[10] K. Moon, A. Szepessy, R. Tempone, G. Zouraris, Convergence rates for adaptive approximation of ordinary differential equations, NUMERISCHE MATHEMATIK 96 (1) (2003) 99-129.

[11] P. Binev, W. Dahmen, R. DeVore, Adaptive finite element methods with convergence rates, NUMERISCHE MATHEMATIK 97 (2) (2004) 219-268.

[12] A. Carpinteri, S. Valente, G. Ferrara, G. Melchiorri, Is mode II fracture energy a real material property?, Comput. Struct. 48 (3) (1993) 397-413.

[13] J. Sarrate, A. Huerta, Efficient unstructured quadrilateral mesh generation., International Journal for Numerical Methods in Engineering 49 (10) (2000) $1327-1350$. 
[14] N. Higham, Accuracy and Stability of Numerical Algorithms, SIAM, Philadelphia, 1996. 